\title{
AN INVERSE PROBLEM IN AMERICAN OPTIONS AS A MATHEMATICAL PROGRAM WITH EQUILIBRIUM CONSTRAINTS: C-STATIONARITY AND AN ACTIVE-SET-NEWTON SOLVER*
}

\author{
MICHAEL HINTERMÜLLER ${ }^{\dagger}$ AND MOULAY HICHAM TBER TH $^{\ddagger}$
}

\begin{abstract}
An inverse problem in the pricing of American options is considered. The problem is formulated as an output least-squares problem governed by a parabolic variational inequality in nondivergence form. The existence of an optimal solution is proved, and first-order optimality conditions of C-stationarity-type are derived by using a relaxation-penalization technique. Numerically, the discrete optimality system is solved by an active-set-Newton solver with feasibility restoration.
\end{abstract}

Key words. American options, parabolic free boundary problem, inverse problem, MPECs, optimality conditions, active-set-Newton solver

AMS subject classifications. 35R35, 35R30, 90C $33,65 \mathrm{~K} 15$

DOI. $10.1137 / 080737277$

1. Introduction. Options are financial instruments that convey the right, but not the obligation, to engage in a future transaction on some underlying asset [40]. There are two major kinds of options: European options that may only be exercised on expiration and American options that may be exercised on any trading day on or before expiration. After the publication of Merton's paper [31] expanding the mathematical understanding of the options pricing model and enhancing the seminal work by Black and Scholes [9], the use of options has been exploited increasingly by investors, corporations, financial institutions, etc. In the Black-Scholes framework, the underlying asset price $S$ is assumed to follow a geometric Brownian motion

$$
d x(t)=\mu x(t) d t+\sigma x(t) d W_{t},
$$

with $W_{t}$ being the increment of a standard Wiener process. The quantities $\mu \in \mathbb{R}$ and $\sigma>0$ are the drift and volatility, respectively. For a European option with maturity date $T$, striking price $K$, and payoff function $P_{0}$, it is possible to show that the option's price at time $t$ is given by the expectation

$$
P(t, x)=\mathbb{E}\left\{e^{-r(T-t)} P_{0}(x(T)) \mid \mathcal{F}_{t}\right\},
$$

where $\mathcal{F}_{t}$ is the natural filtration of $\left(W_{t}\right)_{t}$ and $r$ is the risk-free interest rate. It follows that $P(t, x)$ satisfies the Black-Scholes equation [9]

$$
\frac{\partial P}{\partial t}+\frac{1}{2} \sigma^{2} x^{2} \frac{\partial^{2} P}{\partial x^{2}}+r x \frac{\partial P}{\partial x}-r P=0 .
$$

*Received by the editors October 5, 2008; accepted for publication (in revised form) April 3, 2010; published electronically July 13, 2010.

http://www.siam.org/journals/sicon/48-7/73727.html

${ }^{\dagger}$ Department of Mathematics, Humboldt University of Berlin, Unter den Linden 6, 10099 Berlin, Germany, and START-Project "Interfaces and Free Boundaries," Karl-Franzens University, Department of Mathematics and Scientific Computing, Heinrichstrasse 36, 8010, Graz, Austria (hint@math. hu-berlin.de, michael.hintermueller@uni-graz.at).

${ }_{\ddagger}^{\ddagger}$ Department of Mathematics and Scientific Computing, Karl-Franzens University, Heinrichstrasse 36, 8010, Graz, Austria (moulay.tber@uni-graz.at). 
Due to the possibility of early exercising, the price of an American option is governed by the following optimal stopping time problem:

$$
P(t, x)=\sup _{\text {stopping time } \tau \in[t, T]} \mathbb{E}\left\{e^{-r(\tau-t)} P_{0}(x(\tau)) \mid \mathcal{F}_{t}\right\} .
$$

In this case the price $P(t, x)$ solves the complementarity problem [35]

$$
\min \left\{-\frac{\partial P}{\partial t}-\frac{1}{2} \sigma^{2} x^{2} \frac{\partial^{2} P}{\partial x^{2}}-r x \frac{\partial P}{\partial x}+r P, P-P_{0}\right\}=0 .
$$

Let us mention that the drift $\mu$ was removed from (1.1) and (1.2) by a hedging argument and does not enter explicitly in the model. The strike $K$ enters via the final condition

$$
P(t=T, x)=P_{0}(x)= \begin{cases}\max (x-K, 0) & \text { for call, } \\ \max (K-x, 0) & \text { for put. }\end{cases}
$$

The only parameter not freely observable from the market in (1.1)-(1.3) is the volatility $\sigma$. Therefore gaining information on $\sigma$ is a fundamental issue of option pricing.

Inverse problems in the context of option pricing models have attracted a lot of attention; see, for instance, $[1,2,4,5,6,7,10,11,12,13,14,16,18,19]$ and references therein. However, experience with American options is very limited. To the best of our knowledge, the most complete mathematical and numerical studies in this case are due to Achdou in [1,2] and Achdou and Pironneau in [4]. In those works the local volatility identification problem is formulated as an output least-squares problem. The resulting optimization problem belongs to the problem class of so-called MPECs (mathematical programs with equilibrium constraints), which are hard to handle for several reasons. Indeed, it is well known that complementarity conditions, such as (1.2), occurring as constraints in the minimization problem violate all the known classical NLP (nonlinear programming) constraint qualifications. Hence, the existence of Lagrange multipliers cannot be inferred from standard theory. In $[1,2,4]$, the authors deal with this problem following the penalization approach proposed in [20].

Similarly to [1], in this work we focus on an inverse coefficient problem for American put options. The involved objective functional is different from the objective of the calibration problem. In fact, in our case the objective functional is of distributed form, whereas in the calibration problem a pointwise observation should be considered for different maturities and different strikes. The latter problem requires additional investigations concerning the so-called adjoint system, which is only available in a very weak form and is the subject of ongoing research activities. In this paper, we rather focus on the structure of the first-order necessary conditions and certain sign conditions of the involved Lagrange multipliers; i.e., we focus on the MPEC character of the problem. In fact, similarly to $[1,4]$, we formulate the problem as an output least-squares problem. However, our aim here is to derive first-order optimality conditions of C-stationary-type, which are stronger than the weak-stationarity conditions obtained in $[1,4]$ (for different notions of stationarity for MPECs we refer to $[30,33,34,36,37,41])$. Our approach consists of using first a relaxation technique (cf. [8]) to extend the feasible set of the resulting MPEC and second a Moreau-Yosidabased penalization (cf. [23]) to avoid the low regularity of the Lagrange multiplier corresponding to the state constraint in the relaxed problem. We derive the first-order optimality conditions of the penalized-relaxed subproblems using the classical result 
of Zowe and Kurcyusz [42], and we study the limit for vanishing relaxation parameter and penalty parameter $\gamma \rightarrow+\infty$. We mention here that in contrast to the available work (see, e.g., [32] in the elliptic case), we derive the limit optimality system without considering global solutions of the penalized-relaxed subproblems. In this respect, our approach reflects the typical situation where solution procedures guarantee only stationary points (rather than global minimizers) of nonlinear and nonconvex minimization problems. Moreover, our proof steps motivate numerical algorithms [22]. In the present work, however, based on earlier experience for parameter identification in elliptic variational inequalities [21], we propose an active-set-Newton-type solver for the discrete first-order optimality system of the limit problem.

The organization of the paper is as follows. In section 2 we introduce the forward system and we define its weak solution. The formulation of the inverse problem is given in section 3. In section 4 we study the penalized-relaxed subproblems. Optimality conditions for the original problem are derived in section 5 . We describe in section 6 an active-set-type solver for the corresponding discrete optimality system, and we also provide some numerical results in section 7 . Finally, we discuss in section 8 some extensions of the presented framework - in particular to the local volatility calibration problem.

2. Forward model. We focus on the model for American put options with local volatility $\sigma=\sigma(t, x)$, where $x$ is the underlying asset price and $t$ represents time. Changing $t$ to the time to maturity $T-t$, the option price $P$ of the American put of strike $K$ and maturity $T$ satisfies the complementarity system

$$
\begin{aligned}
\mathcal{L}_{B S}(P(t, x)) & \geq 0, & & (t, x) \in(0, T] \times \Omega, \\
\mathcal{L}_{B S}(P(t, x)) \cdot\left(P(t, x)-P_{0}(x)\right) & =0, & & (t, x) \in(0, T] \times \Omega, \\
P(t, x)-P_{0}(x) & \geq 0, & & (t, x) \in(0, T] \times \Omega, \\
P(t, x)-P_{0}(x) & =0, & & (t, x) \in(0, T] \times \partial \Omega, \\
P(t=0, x)-P_{0}(x) & =0, & & x \in \Omega,
\end{aligned}
$$

where

$$
\mathcal{L}_{B S}(P(t, x))=\frac{\partial P(t, x)}{\partial t}-\frac{\sigma^{2}(t, x) x^{2}}{2} \frac{\partial^{2} P(t, x)}{\partial x^{2}}-r x \frac{\partial P(t, x)}{\partial x}+r P(t, x)
$$

and the payoff function $P_{0}(x)$ is defined by

$$
P_{0}(x)=(K-x)^{+}=\max (0, K-x) .
$$

Here we consider a truncated domain in $x$; i.e., for $\bar{x}$ large enough, particularly larger than $K$, we consider $\Omega=(\underline{x}, \bar{x})$ as the spatial domain and $Q=(0, T) \times \Omega$ as the time-space domain. The positive real $\underline{x}$ is chosen such that $P(t, x)$ coincides with $P_{0}(x)$ in the rectangle $[0, T] \times[0, \underline{x}]$. Due to the result given in $[1, \mathrm{Thm} .3 .4]$ (see also [4, p. 206]), such a choice is possible for the family of volatilities considered in the next sections. For the error inflicted by introducing the upper bound $\bar{x}$ we refer to $[26]$.

To streamline the presentation, we restrict ourselves to the case of zero interest, i.e. $r=0$, and, for some $\tau>0$, we introduce the new variables

$$
\begin{aligned}
y_{0}(t, x) & =e^{-\tau t} P_{0}(x), \\
y(t, x) & =e^{-\tau t}\left(P(t, x)-P_{0}(x)\right) .
\end{aligned}
$$


We further denote

$$
\begin{aligned}
u(t, x) & =\frac{\sigma(t, x)^{2} x^{2}}{2}, \\
f(u) & =u \frac{\partial^{2} y_{0}}{\partial x^{2}}, \\
\mathcal{A}(u) y & =-u \frac{\partial^{2} y}{\partial x^{2}}+\tau y, \\
\mathcal{A}^{\prime}(u) y & =-u \frac{\partial^{2} y}{\partial x^{2}} .
\end{aligned}
$$

With these transformations, the forward model reads

$$
\left\{\begin{aligned}
\frac{\partial y}{\partial t}+\mathcal{A}(u) y-f(u) & \geq 0 & & \text { in } Q \\
\left(\frac{\partial y}{\partial t}+\mathcal{A}(u) y-f(u)\right) y & =0 & & \text { in } Q \\
y & =0 & & \text { in }(0, T) \times \partial \Omega \\
y(0) & =0 & & \text { in } \Omega \\
y & \geq 0 & & \text { in } Q .
\end{aligned}\right.
$$

For the variational formulation of (2.14) we introduce the Hilbert space

$$
W(0, T)=\left\{v \in L^{2}\left(0, T, H_{0}^{1}(\Omega)\right): \frac{\partial v}{\partial t} \in L^{2}\left(0, T, H^{-1}(\Omega)\right)\right\}
$$

and the subset

$$
\mathcal{K}=\{v \in W(0, T): v \geq 0 \text { a.e. in } Q\} .
$$

Here and below, "a.e." stands for "almost everywhere."

A function $y(t, x)$ is then called a weak solution of (2.14) if

$$
\left\{\begin{aligned}
y & \in \mathcal{K} \\
\left\langle\frac{\partial y}{\partial t}+\mathcal{A}(u) y-f(u), v-y\right\rangle & \geq 0 \quad \forall v \in \mathcal{K}, \\
y(0) & =0 \quad \text { a.e. in } \Omega
\end{aligned}\right.
$$

where $\langle\cdot, \cdot\rangle$ is the duality product between $L^{2}\left(0, T, H^{-1}(\Omega)\right)$ and $L^{2}\left(0, T, H_{0}^{1}(\Omega)\right)$. We recall that for $u$ in $L^{\infty}\left(0, T, H^{1}(\Omega)\right)$, and $v$ and $w$ in $L^{2}\left(0, T, H_{0}^{1}(\Omega)\right)$, we have

$$
\begin{gathered}
\langle\mathcal{A}(u) v, w\rangle=\int_{0}^{T} \int_{\Omega} u \frac{\partial v}{\partial x} \frac{\partial w}{\partial x} d x d t+\int_{0}^{T} \int_{\Omega} \frac{\partial u}{\partial x} \frac{\partial v}{\partial x} w d x d t+\tau \int_{0}^{T} \int_{\Omega} v w d x d t \\
-\langle f(u), w\rangle=\int_{0}^{T} \int_{\Omega} u \frac{\partial y_{0}}{\partial x} \frac{\partial w}{\partial x} d x d t+\int_{0}^{T} \int_{\Omega} \frac{\partial u}{\partial x} \frac{\partial y_{0}}{\partial x} w d x d t
\end{gathered}
$$

and

$$
\left\langle\mathcal{A}^{\prime}(u) v, w\right\rangle=\int_{0}^{T} \int_{\Omega} u \frac{\partial v}{\partial x} \frac{\partial w}{\partial x} d x d t+\int_{0}^{T} \int_{\Omega} \frac{\partial u}{\partial x} \frac{\partial v}{\partial x} w d x d t
$$

Moreover, we can easily show the following properties.

Copyright $\odot$ by SIAM. Unauthorized reproduction of this article is prohibited. 
LEMMA 2.1.

(i) For all $u \in L^{\infty}\left(0, T, W^{1, \infty}(\Omega)\right)$ and $y \in L^{2}\left(0, T, H_{0}^{1}(\Omega)\right)$ we have

$$
\begin{aligned}
\|\mathcal{A}(u) y\|_{L^{2}\left(0, T, H^{-1}(\Omega)\right)} & \leq\left(\|u\|_{L^{\infty}\left(0, T, W^{1, \infty}(\Omega)\right)}+\tau\right)\|y\|_{L^{2}\left(0, T, H^{1}(\Omega)\right)}, \\
\|f(u)\|_{L^{2}\left(0, T, H^{-1}(\Omega)\right)} & \leq\|u\|_{L^{\infty}\left(0, T, W^{1, \infty}(\Omega)\right)}\left\|y_{0}\right\|_{L^{2}\left(0, T, H_{0}^{1}(\Omega)\right)}, \\
\left\|\mathcal{A}^{\prime}(u) y\right\|_{L^{2}\left(0, T, H^{-1}(\Omega)\right)} & \leq\|u\|_{L^{\infty}\left(0, T, W^{1, \infty}(\Omega)\right)}\|y\|_{L^{2}\left(0, T, H^{1}(\Omega)\right)} .
\end{aligned}
$$

(ii) Let $\left(u_{k}, y_{k}, z_{k}\right)_{k \geq 0}$ be a sequence such that

$$
\begin{array}{ll}
u_{k} \longrightarrow u & \text { in } L^{\infty}\left(0, T, W^{1, \infty}(\Omega)\right), \\
y_{k} \longrightarrow y & \text { in } L^{2}\left(0, T, H_{0}^{1}(\Omega)\right), \\
z_{k} \rightarrow z & \text { in } L^{2}\left(0, T, H_{0}^{1}(\Omega)\right),
\end{array}
$$

where $\longrightarrow$ denotes strong convergence and $\rightarrow$ weak convergence. Then

$$
\begin{aligned}
& f\left(u_{k}\right) \longrightarrow f(u) \quad \text { in } L^{2}\left(0, T, H^{-1}(\Omega)\right), \\
& \mathcal{A}^{\prime}\left(u_{k}\right) y_{k} \longrightarrow \mathcal{A}^{\prime}(u) y \quad \text { in } L^{2}\left(0, T, H^{-1}(\Omega)\right) \text {, } \\
& \mathcal{A}\left(u_{k}\right) y_{k} \longrightarrow \mathcal{A}(u) y \quad \text { in } L^{2}\left(0, T, H^{-1}(\Omega)\right) \text {, } \\
& \mathcal{A}\left(u_{k}\right) z_{k} \rightarrow \mathcal{A}(u) z \quad \text { in } L^{2}\left(0, T, H^{-1}(\Omega)\right) \text {. }
\end{aligned}
$$

(iii) For $\tau$ large enough, and under the assumption

$$
\left\{\begin{array}{cc}
\text { there exist } \underline{u}, \bar{u}, \text { and } M & \text { such that } \\
0<\underline{u} \leq u(t, x) \leq \bar{u} & \text { a.e. in } Q \\
\left|\frac{\partial u(t, x)}{\partial x}\right| \leq M & \text { a.e. in } Q
\end{array}\right.
$$

there exist positive constants $C_{f}, C_{\mathcal{A}^{\prime}}, C_{\mathcal{A}}$, and $\omega$ not depending on $u$ or $y$ such that

$$
\begin{aligned}
\|f(u)\|_{L^{2}\left(0, T, H^{-1}(\Omega)\right)} & \leq C_{f}, \\
\left\|\mathcal{A}^{\prime}(u) y\right\|_{L^{2}\left(0, T, H^{-1}(\Omega)\right)} & \leq C_{\mathcal{A}^{\prime}}\|y\|_{L^{2}\left(0, T, H_{0}^{1}(\Omega)\right)}, \\
\|\mathcal{A}(u) y\|_{L^{2}\left(0, T, H^{-1}(\Omega)\right)} & \leq C_{\mathcal{A}}\|y\|_{L^{2}\left(0, T, H_{0}^{1}(\Omega)\right)}, \\
\langle\mathcal{A}(u) y, y\rangle & \geq \omega\|y\|_{L^{2}\left(0, T, H_{0}^{1}(\Omega)\right)}^{2}
\end{aligned}
$$

(iv) The function $y$ is a weak solution of (2.14) if and only if $P(c f .(2.9))$ is a solution of the following nonhomogeneous variational inequality problem:

$(\mathrm{NVI})$

$$
\left\{\begin{array}{l}
\text { Find } P \in \mathcal{K}\left(P_{0}\right) \text { such that } \\
\left\langle\frac{\partial P}{\partial t}-u \frac{\partial^{2} P}{\partial x^{2}}, S-P\right\rangle \geq 0 \quad \forall S \in \mathcal{K}\left(P_{0}\right), \\
P(0)=P_{0} \quad \text { a.e. in } Q,
\end{array}\right.
$$

with

$$
\begin{gathered}
\mathcal{K}\left(P_{0}\right)=\left\{S \in L^{2}\left(0, T, H^{1}(\Omega)\right): \frac{\partial S}{\partial t} \in L^{2}\left(0, T, H^{-1}(\Omega)\right),\right. \\
\left.S \geq P_{0} \text { a.e. in } Q, S=P_{0} \text { in }(0, T] \times \partial \Omega\right\} .
\end{gathered}
$$

Copyright $@$ by SIAM. Unauthorized reproduction of this article is prohibited. 
Now we can state the existence, uniqueness, and regularity result. For its proof we refer to [1, Thm. 2.2] or [4].

THEOREM 2.2. Under assumption (A1), there exists a unique solution $P$ to the nonhomogeneous problem (NVI). The solution $P$ is such that $\frac{\partial P}{\partial t}$ and $\frac{\partial^{2} P}{\partial x^{2}}$ are in $L^{2}(Q)$. Furthermore, there exists a constant $C$ depending only on $Q, \underline{u}, \bar{u}, M$, and $K$ such that

$$
\begin{aligned}
\|P\|_{L^{2}\left(0, T, H^{1}(\Omega)\right)} & \leq C, \\
\left\|\frac{\partial^{2} P}{\partial x^{2}}\right\|_{L^{2}(Q)} & \leq C, \\
\left\|\frac{\partial P}{\partial t}\right\|_{L^{2}(Q)} & \leq C .
\end{aligned}
$$

COROLlaRY 2.3. Under assumption (A1), the complementarity problem (2.14) admits a unique weak solution $y$. Moreover, there exist a Lagrange multiplier $\xi$ in $L^{2}(Q)$ and a constant $C$ depending only on $Q, \underline{u}, \bar{u}, M$, and $K$ such that

$$
\begin{cases}\frac{\partial y}{\partial t}+\mathcal{A}(u) y-f(u)=\xi & \text { a.e. in } Q \\ y(0)=0 & \text { a.e. in } \Omega, \\ \xi \geq 0 \text { a.e. in } Q, \quad y \geq 0 & \text { a.e. in } Q, \quad(\xi, y)_{L^{2}(Q)}=0\end{cases}
$$

and

$$
\begin{aligned}
\|y\|_{W(0, T)} & \leq C \\
\|\xi\|_{L^{2}(Q)} & \leq C
\end{aligned}
$$

Proof. The existence and uniqueness of the weak solution $y$ of (2.14) as well as the boundedness of $y$ in $W(0, T)$ are immediate consequences of the previous theorem and Lemma 2.1(iv). Moreover, by (2.9), we have

$$
\frac{\partial y}{\partial t}+\mathcal{A}(u) y-f(u)=e^{-\tau t}\left(\frac{\partial P}{\partial t}-u \frac{\partial^{2} P}{\partial x^{2}}\right) \text { in } L^{2}\left(0, T, H^{-1}(\Omega)\right),
$$

where $P$ is the solution of (NVI). Hence, if we set

$$
\xi=e^{-\tau t}\left(\frac{\partial P}{\partial t}-u \frac{\partial^{2} P}{\partial x^{2}}\right)
$$

the existence of the Lagrange multiplier and its boundedness in $L^{2}(Q)$ follow from assumption (A1), (2.25), and (2.26). The nonnegativity of $\xi$ can be deduced by choosing $S=P+\phi$ in (NVI) with arbitrary $\phi \in W(0, T), \phi \geq 0$.

Copyright $\odot$ by SIAM. Unauthorized reproduction of this article is prohibited. 
3. Least-squares formulation. In view of Corollary 2.3, we formulate the inverse coefficient problem as the following least-squares problem:

$\left(\mathrm{P}_{0}\right)$

$$
\begin{cases}\min & J(y, u)=\frac{1}{2}\left\|y-y_{d}\right\|_{L^{2}(Q)}^{2}+\frac{1}{2}\left\|y(T, \cdot)-y_{T}\right\|_{L^{2}(\Omega)}^{2}+\frac{\nu}{2}\|u\|_{\mathcal{U}}^{2} \\ \text { over } & (y, u, \xi) \in W(0, T) \times \mathcal{U} \times L^{2}(Q) \\ \text { s.t. (subject to }) & u \in U_{a d}=\left\{u \in \mathcal{U}: 0<\underline{u} \leq u \leq \bar{u} \text { and }\left|\frac{\partial u}{\partial x}\right| \leq M \text { a.e. in } Q\right\}, \\ & \frac{\partial y}{\partial t}+\mathcal{A}(u) y-f(u)-\xi=0 \text { in } L^{2}\left(0, T, H^{-1}(\Omega)\right), \\ & y(0)=0 \text { a.e. in } \Omega, \\ & y \geq 0 \text { a.e. in } Q, \quad \xi \geq 0 \text { a.e. in } Q, \quad(\xi, y)_{L^{2}(Q)}=0,\end{cases}
$$

with given data $y_{d} \in L^{2}(Q)$ and $y_{T} \in L^{2}(\Omega)$. The quantities $\nu, \underline{u}, \bar{u}$, and $M$ are positive real numbers with $\underline{u}<\bar{u}$. Further, $U_{a d}$ is a closed convex subset of the Hilbert space $\mathcal{U}=H^{1}\left(0, T, H^{2}(\Omega)\right)$ supplied with the inner product

$$
\begin{aligned}
& \langle u, v\rangle_{\mathcal{U}}=\quad(u, v)_{L^{2}(Q)}+\left(\frac{\partial u}{\partial t}, \frac{\partial v}{\partial t}\right)_{L^{2}(Q)} \\
& +\left(\frac{\partial u}{\partial x}, \frac{\partial v}{\partial x}\right)_{L^{2}(Q)}+\left(\frac{\partial^{2} u}{\partial t \partial x}, \frac{\partial^{2} v}{\partial t \partial x}\right)_{L^{2}(Q)} \\
& +\left(\frac{\partial^{2} u}{\partial x^{2}}, \frac{\partial^{2} v}{\partial x^{2}}\right)_{L^{2}(Q)}+\left(\frac{\partial^{3} u}{\partial t \partial x^{2}}, \frac{\partial^{3} v}{\partial t \partial x^{2}}\right)_{L^{2}(Q)}
\end{aligned}
$$

and the induced norm

$$
\|u\|_{\mathcal{U}}^{2}=\langle u, u\rangle_{\mathcal{U}}
$$

The key compactness results needed in this paper are given by the following lemma.

LEMMA 3.1.

(1) The space $W(0, T)$ is continuously embedded in $\mathcal{C}^{0}\left(0, T, L^{2}(\Omega)\right)$ and compactly embedded in $L^{2}(Q)$.

(2) The space $\mathcal{U}$ is continuously and compactly embedded in $L^{\infty}\left(0, T, W^{1, \infty}(\Omega)\right)$.

Proof. Assertion (1) is a classical result whose proof can be found, for instance, in [38]. For the proof of (2) let us consider the space $\mathcal{W}$ defined by

$$
\mathcal{W}=\left\{u \in L^{2}(Q): \frac{\partial u}{\partial x}, \frac{\partial u}{\partial t}, \frac{\partial^{2} u}{\partial t \partial x} \in L^{2}(Q)\right\}
$$

According to [29, Lem. 2.1], $\mathcal{W}$ embeds continuously and compactly in $L^{\infty}(0, T$, $\left.L^{\infty}(\Omega)\right)$. Moreover, it is clear that

$$
H^{1}\left(0, T, H^{2}(\Omega)\right)=\left\{u \in \mathcal{W}: \frac{\partial u}{\partial x} \in \mathcal{W}\right\} .
$$

Hence $\mathcal{U}$ embeds continuously and compactly in $L^{\infty}\left(0, T, W^{1, \infty}(\Omega)\right)$.

The following proposition will be useful for establishing the next results. In view of Lemma 2.1(iii), henceforth $\tau$ is chosen large enough such that (2.20)-(2.23) hold for any $y \in L^{2}\left(0, T, H_{0}^{1}(\Omega)\right)$ and $u \in U_{a d}$.

Copyright $@$ by SIAM. Unauthorized reproduction of this article is prohibited. 
Proposition 3.2. Let $\left(y_{k}, u_{k}, \xi_{k}\right)_{k \geq 0}$ be a sequence in $W(0, T) \times U_{a d} \times L^{2}(Q)$ such that $\left(u_{k}\right)_{k \geq 0}$ is bounded in $U_{a d},\left(\bar{\xi}_{k}\right)_{k \geq 0}$ is bounded in $L^{2}(Q)$, and $y_{k}$ is the solution of the parabolic equation

$$
\begin{array}{rlll}
\frac{\partial y_{k}}{\partial t}+\mathcal{A}\left(u_{k}\right) y_{k}-f\left(u_{k}\right)-\xi_{k} & =0 & \text { in } L^{2}\left(0, T, H^{-1}(\Omega)\right), \\
y_{k}(0) & =0 & \text { a.e. in } \Omega .
\end{array}
$$

Then there exist $(y, u, \xi)$ in $W(0, T) \times U_{a d} \times L^{2}(Q)$ and a subsequence still denoted by $\left(y_{k}, u_{k}, \xi_{k}\right)_{k \geq 0}$ such that

$$
\begin{aligned}
u_{k} \rightarrow u & \text { in } \mathcal{U} \\
\xi_{k} \rightarrow \xi & \text { in } L^{2}(Q), \\
y_{k} \rightarrow y & \text { in } W(0, T), \\
u_{k} \rightarrow u & \text { in } L^{\infty}\left(0, T, W^{1, \infty}(\Omega)\right), \\
y_{k} \rightarrow y & \text { in } L^{2}(Q),
\end{aligned}
$$

and

$$
\begin{array}{rlll}
\frac{\partial y}{\partial t}+\mathcal{A}(u) y-f(u)-\xi & = & & \text { in } L^{2}\left(0, T, H^{-1}(\Omega)\right), \\
y(0) & = & 0 & \text { a.e. in } \Omega .
\end{array}
$$

Furthermore,

$$
\begin{aligned}
& y_{k} \longrightarrow y \quad \text { in } L^{2}\left(0, T, H_{0}^{1}(\Omega)\right), \\
& y_{k}(T) \longrightarrow y(T) \quad \text { in } L^{2}(Q) \text {. }
\end{aligned}
$$

Proof. By testing (3.2) against $y_{k}$ and $\phi \in L^{2}\left(0, T, H_{0}^{1}(\Omega)\right)$, and using Lemma 2.1(iii) we obtain

$$
\begin{aligned}
\omega\left\|y_{k}\right\|_{L^{2}\left(0, T, H_{0}^{1}(\Omega)\right)}^{2} \leq & C_{f}\left\|y_{k}\right\|_{L^{2}\left(0, T, H_{0}^{1}(\Omega)\right)}+\left\|\xi_{k}\right\|_{L^{2}(Q)}\left\|y_{k}\right\|_{L^{2}(Q)} \\
\left\langle\frac{\partial y_{k}}{\partial t}, \phi\right\rangle \leq & C_{\mathcal{A}}\left\|y_{k}\right\|_{L^{2}\left(0, T, H_{0}^{1}(\Omega)\right)}\|\phi\|_{L^{2}\left(0, T, H_{0}^{1}(\Omega)\right)} \\
& +C_{f}\|\phi\|_{L^{2}\left(0, T, H_{0}^{1}(\Omega)\right)}+\left\|\xi_{k}\right\|_{L^{2}(Q)}\|\phi\|_{L^{2}(Q)}
\end{aligned}
$$

Taking into account the boundedness of $\left(\xi_{k}\right)_{k \geq 0}$ in $L^{2}(Q)$, we deduce that $\left(y_{k}\right)_{k \geq 0}$ is bounded in $W(0, T)$. Therefore,

$$
\left(y_{k}, u_{k}, \xi_{k}\right)_{k \geq 0} \text { is bounded in } W(0, T) \times \mathcal{U} \times L^{2}(Q)
$$

and (3.3)-(3.5) follow. The assertions (3.6)-(3.7) are immediate consequences of (3.13) and Lemma 3.1. Due to Lemma 2.1(ii), the limit element $(y, u, \xi) \in W(0, T) \times \mathcal{U} \times$ $L^{2}(Q)$ satisfies

$$
\frac{\partial y}{\partial t}+\mathcal{A}(u) y-f(u)-\xi=0 \quad \text { in } L^{2}\left(0, T, H^{-1}(\Omega)\right) .
$$

Now let $\varphi$ be an arbitrary element of $H_{0}^{1}(\Omega)$, and let $\phi \in \mathcal{C}^{1}(0, T)$ such that $\phi(0) \neq 0$ and $\phi(T)=0$. Multiplying (3.2) by $\psi=\varphi \phi$ and using integration by parts yield

$$
-\left\langle y_{k}, \frac{\partial \psi}{\partial t}\right\rangle+\left\langle\mathcal{A}\left(u_{k}\right) y_{k}-f\left(u_{k}\right)-\xi_{k}, \psi\right\rangle=0 .
$$

Copyright $@$ by SIAM. Unauthorized reproduction of this article is prohibited. 
Passing to the limit as $k \longrightarrow \infty$ we obtain

$$
-\left\langle y, \frac{\partial \psi}{\partial t}\right\rangle+\langle\mathcal{A}(u) y-f(u)-\xi, \psi\rangle=0
$$

Integration by parts and (3.14) yield

$$
\int_{\Omega} y(0, x) \psi(0, x) d x=0,
$$

which implies

$$
\int_{\Omega} y(0, x) \varphi(x) d x=0 .
$$

As $\varphi$ was chosen arbitrarily in $H_{0}^{1}(\Omega)$, we deduce

$$
y(0)=0 .
$$

For the strong convergence of $y_{k}$ and $y_{k}(T)$, respectively, we subtract (3.8) from (3.2) and multiply by $y_{k}-y$. This yields

$$
\begin{aligned}
\frac{1}{2}\left\|y(T)-y_{k}(T)\right\|_{L^{2}(\Omega)}^{2}+\omega\left\|y-y_{k}\right\|_{L^{2}\left(0, T, H_{0}^{1}(\Omega)\right)}^{2} \leq & \left\langle\left(\mathcal{A}\left(u_{k}\right)-\mathcal{A}(u)\right) y, y-y_{k}\right\rangle \\
& +\left\langle f\left(u_{k}\right)-f(u), y-y_{k}\right\rangle \\
& +\left(\xi_{k}-\xi, y-y_{k}\right)_{L^{2}(Q)} .
\end{aligned}
$$

As $u_{k}$ converges to $u$ in $L^{\infty}\left(0, T, W^{1, \infty}(\Omega)\right)$, we deduce again from Lemma 2.1(ii) that

$$
\begin{aligned}
f\left(u_{k}\right) & \longrightarrow f(u) \quad \text { in } L^{2}\left(0, T, H^{-1}(\Omega)\right), \\
\mathcal{A}\left(u_{k}\right) y & \longrightarrow \mathcal{A}(u) y \text { in } L^{2}\left(0, T, H^{-1}(\Omega)\right) .
\end{aligned}
$$

This, together with (3.4), (3.5), and (3.7), implies that the right-hand side of (3.16) vanishes as $k \longrightarrow 0$. Consequently (3.9)-(3.10) hold true.

THEOREM 3.3. The problem $\left(\mathrm{P}_{0}\right)$ has at least one solution.

Proof. Let $\left(y_{k}, u_{k}, \xi_{k}\right)_{k \geq 1}$ be a minimizing sequence for $\left(\mathrm{P}_{0}\right)$ such that

$$
\inf \left(\mathrm{P}_{0}\right) \leq \frac{1}{2}\left\|y_{k}-y_{d}\right\|_{L^{2}(Q)}^{2}+\frac{1}{2}\left\|y_{k}(T, \cdot)-y_{T}\right\|_{L^{2}(\Omega)}^{2}+\frac{\nu}{2}\left\|u_{k}\right\|_{\mathcal{U}}^{2} \leq \inf \left(\mathrm{P}_{0}\right)+\frac{1}{k} .
$$

Then $\left(u_{k}\right)_{k \geq 1}$ is bounded in $U_{a d}$ and, by $(2.28),\left(\xi_{k}\right)_{k \geq 1}$ is bounded in $L^{2}(Q)$. Hence, by Proposition 3.2, there exists $(y, u, \xi)$ in $W(0, T) \times U_{a d} \times L^{2}(Q)$ and a subsequence still denoted by $\left(y_{k}, u_{k}, \xi_{k}\right)_{k \geq 1}$ such that (3.3)-(3.10) hold. Moreover, we can easily check that $(y, u, \xi)$ satisfies

$$
y \geq 0 \text { a.e. in } Q, \quad \xi \geq 0 \text { a.e. in } Q, \quad \text { and }(\xi, y)_{L^{2}(Q)}=0,
$$

which implies that $(y, u, \xi)$ is a feasible point for $\left(\mathrm{P}_{0}\right)$.

On the other hand, (3.3), (3.7), (3.10), and the weak lower semicontinuity of norms yield

$$
J(y, u) \leq \liminf _{k \rightarrow \infty} J\left(y_{k}, u_{k}\right) \leq \inf \left(\mathrm{P}_{0}\right) .
$$

Copyright (C) by SIAM. Unauthorized reproduction of this article is prohibited. 
Consequently $(y, u, \xi)$ is an optimal solution of $\left(\mathrm{P}_{0}\right)$.

From now on, we add an explicit constraint to $\left(\mathrm{P}_{0}\right)$ involving the multiplier $\xi$ in $L^{2}(Q)$. The new least-squares formulation reads

$$
\begin{cases}\min & J(y, u) \\ \text { over } & (y, u, \xi) \in W(0, T) \times \mathcal{U} \times L^{2}(Q) \\ \text { s.t. } & u \in U_{a d} \\ & \frac{\partial y}{\partial t}+\mathcal{A}(u) y-f(u)-\xi=0 \quad \text { in } L^{2}\left(0, T, H^{-1}(\Omega)\right), \\ & y(0)=0 \text { in } \Omega, \\ & y \geq 0 \text { a.e. in } Q, \quad \xi \geq 0 \text { a.e. in } Q, \quad(\xi, y)_{L^{2}(Q)}=0 \\ & \frac{1}{2}\|\xi\|_{L^{2}(Q)}^{2} \leq R\end{cases}
$$

where $R$ is a sufficiently large positive constant. For instance, we choose $R$ such that

$$
R \geq \frac{1}{2} C^{2}
$$

with $C$ being the constant from (2.28). For this choice of $R$, the new constraint is redundant. However, as $(\mathrm{P})$ lacks constraint regularity, for deriving stationarity conditions for $\left(\mathrm{P}_{0}\right)$ in the next section we relax the constraints of $(\mathrm{P})$ such that the relaxed version of $(\mathrm{P})$ satisfies well-known constraint qualifications of mathematical programming in Banach spaces [42]. In this context, it turns out that the wellposedness of the relaxed version of $(\mathrm{P})$ depends on the new constraint for $\xi$ [8].

4. Penalized-relaxed problem. In this section we introduce and study a penalized-relaxed version of the optimal control problem (P). Following the approach in [22], our objective is to characterize some type of C-stationarity of critical points of $(\mathrm{P})$. This is achieved by passing to the limit with respect to the penalization and relaxation parameters. The penalized-relaxed problem is defined as follows:

$$
\left(\mathrm{P}_{\gamma}\right) \quad \begin{cases}\min & J_{\gamma}(y, u)=J(y, u)+\frac{1}{2 \gamma}\|\max (0, \bar{\lambda}-\gamma y)\|_{L^{2}(\Omega)}^{2} \\ \text { over } \quad & (y, u, \xi) \in W(0, T) \times \mathcal{U} \times L^{2}(Q) \\ \text { s.t. } \quad & u \in U_{a d}, \\ & \frac{\partial y}{\partial t}+\mathcal{A}(u) y-f(u)-\xi=0 \quad \text { in } L^{2}\left(0, T, H^{-1}(\Omega)\right), \\ & y(0)=0 \text { in } \Omega, \\ & \xi \geq 0 \text { a.e. in } Q, \quad(\xi, y)_{L^{2}(Q)} \leq \alpha_{\gamma}, \\ & R \geq \frac{1}{2}\|\xi\|_{L^{2}(Q)}^{2},\end{cases}
$$

where $\bar{\lambda} \in L^{2}(Q)$, which mimics a regular version of the multiplier associated to $y \geq 0$, is arbitrarily fixed with $\bar{\lambda} \geq 0$ a.e. in $Q$. Note that we add the penalty term $\frac{1}{2 \gamma}\|\max (0, \bar{\lambda}-\gamma y)\|_{L^{2}(\Omega)}^{2}$ to $J(y, u)$ with $\gamma$ denoting the associated penalty parameter. This step relaxes the pointwise state constraint $y \geq 0$ a.e. in $Q$. The derivative of the penalty term serves as a regular (i.e., $\left.L^{2}(Q)\right)$ approximation of the multiplier associated with $y \geq 0$ a.e. in $Q$. Further, we $\operatorname{relax}(\xi, y)_{L^{2}(Q)}=0$ by allowing $(\xi, y)_{L^{2}(Q)} \leq \alpha_{\gamma}$ for some $\alpha_{\gamma}>0$. These modifications motivate the description 
of $\left(\mathrm{P}_{\gamma}\right)$ as the penalized-relaxed version of $(\mathrm{P})$. Subsequently we are interested in $\gamma \rightarrow \infty$ and $\alpha_{\gamma} \downarrow 0$ as $\gamma \rightarrow \infty$.

Let $\mathcal{D}_{\gamma}$ and $\mathcal{D}$ denote the feasible sets of $\left(\mathrm{P}_{\gamma}\right)$ and $(\mathrm{P})$, respectively. Observe that we have

$$
\mathcal{D}_{\gamma} \supseteq \mathcal{D} \neq \emptyset
$$

\subsection{Global solutions.}

TheOREM 4.1. For every $\gamma>0$, the penalized-relaxed problem $\left(\mathrm{P}_{\gamma}\right)$ possesses at least one solution $\left(y_{\gamma}, u_{\gamma}, \xi_{\gamma}\right)$. Furthermore, there exist $\left(y^{*}, u^{*}, \xi^{*}\right)$ in $W(0, T) \times \mathcal{U} \times$ $L^{2}(Q)$ and a subsequence still denoted by $\left(y_{\gamma}, u_{\gamma}, \xi_{\gamma}\right)_{\gamma>0}$ such that

$$
\begin{array}{cl}
u_{\gamma} \rightarrow u^{*} & \text { in } \mathcal{U}, \\
\xi_{\gamma} \rightarrow \xi^{*} & \text { in } L^{2}(Q), \\
y_{\gamma} \rightarrow y^{*} & \text { in } W(0, T), \\
u_{\gamma} \longrightarrow u^{*} & \text { in } L^{\infty}\left(0, T, W^{1, \infty}(\Omega)\right), \\
y_{\gamma} \longrightarrow y^{*} & \text { in } L^{2}\left(0, T, H_{0}^{1}(\Omega)\right), \\
y_{\gamma}(T) \longrightarrow y^{*}(T) & \text { in } L^{2}(Q),
\end{array}
$$

and $\left(y^{*}, u^{*}, \xi^{*}\right)$ is a solution of $(\mathrm{P})$.

Proof. The existence of a solution for $\left(\mathrm{P}_{\gamma}\right)$ follows from the weak lower semicontinuity of $J_{\gamma}$ and Proposition 3.2 applied to a minimizing sequence.

For the convergence of $\left(y_{\gamma}, u_{\gamma}, \xi_{\gamma}\right)_{\gamma>0}$, let $(\tilde{y}, \tilde{u}, \tilde{\xi}) \in \mathcal{D}$ and $\gamma \geq 1$. We have

$$
J_{\gamma}\left(y_{\gamma}, u_{\gamma}\right) \leq J_{\gamma}(\tilde{y}, \tilde{u}) \leq J(\tilde{y}, \tilde{u})+\frac{1}{2}\|\max (0, \bar{\lambda})\|^{2} .
$$

Hence

$$
\begin{aligned}
&\left(\xi_{\gamma}\right)_{\gamma \geq 1} \text { is bounded in } L^{2}(Q), \\
&\left(u_{\gamma}\right)_{\gamma \geq 1} \text { is bounded in } \mathcal{U}, \\
&\left(\frac{1}{\sqrt{\gamma}} \max \left(0, \bar{\lambda}-\gamma y_{\gamma}\right)\right)_{\gamma \geq 1} \text { is bounded in } L^{2}(Q) .
\end{aligned}
$$

From (4.8), (4.9), and Proposition 3.2 we deduce the existence of $\left(y^{*}, u^{*}, \xi^{*}\right) \in W(0, T)$ $\times \mathcal{U} \times L^{2}(Q)$ and a subsequence still denoted by $\left(y_{\gamma}, u_{\gamma}, \xi_{\gamma}\right)_{\gamma \geq 1}$ such that

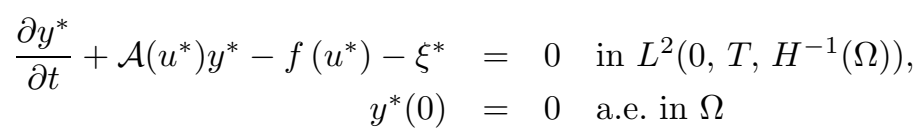

and (4.1)-(4.6) hold. It follows also that

$$
\begin{aligned}
\xi^{*} & \geq 0 \quad \text { a.e. in } Q, \\
\left(\xi^{*}, y^{*}\right)_{L^{2}(Q)} & \leq 0, \\
R & \geq \frac{1}{2}\left\|\xi^{*}\right\|_{L^{2}(Q)}^{2} .
\end{aligned}
$$

Moreover, we have $y^{*} \geq 0$ a.e. in $Q$. Indeed,

$$
\frac{1}{\gamma}\left\|\max \left(0, \bar{\lambda}-\gamma y_{\gamma}\right)\right\|_{L^{2}(Q)}=\left\|\max \left(0, \frac{\bar{\lambda}}{\gamma}-y_{\gamma}\right)\right\|_{L^{2}(Q)} \longrightarrow 0
$$

Copyright (c) by SIAM. Unauthorized reproduction of this article is prohibited. 
from (4.10). On the other hand, by (4.5) and Lebesgue's dominated convergence theorem we obtain

$$
\left\|\max \left(0, \frac{\bar{\lambda}}{\gamma}-y_{\gamma}\right)\right\|_{L^{2}(Q)} \longrightarrow\left\|\max \left(0,-y^{*}\right)\right\|_{L^{2}(Q)} .
$$

Consequently,

$$
\left\|\max \left(0,-y^{*}\right)\right\|_{L^{2}(Q)}=0
$$

and

$$
y^{*} \geq 0 \text { a.e. in } Q .
$$

This, with (4.12) and (4.13), implies

$$
\left(y^{*}, \xi^{*}\right)_{L^{2}(Q)}=0 .
$$

Now for all $(y, u, \xi) \in \mathcal{D} \subseteq \mathcal{D}_{\gamma}$, we have

$$
J(y, u)=\lim _{\gamma \longrightarrow \infty} J_{\gamma}(y, u) \geq \liminf _{\gamma \longrightarrow \infty} J_{\gamma}\left(y_{\gamma}, u_{\gamma}\right) \geq \liminf _{\gamma \longrightarrow \infty} J\left(y_{\gamma}, u_{\gamma}\right) .
$$

From (4.1), (4.5), (4.6), and the weak lower semicontinuity of $J$ we infer

$$
\liminf _{\gamma \longrightarrow \infty} J\left(y_{\gamma}, u_{\gamma}\right) \geq J\left(y^{*}, u^{*}\right) .
$$

Hence

$$
J(y, u) \geq J\left(y^{*}, u^{*}\right) \quad \forall(y, u) \in \mathcal{D} .
$$

From $(4.11),(4.12),(4.14),(4.18),(4.19)$, and (4.20) we deduce that $\left(y^{*}, u^{*}, \xi^{*}\right)$ is a solution of $(\mathrm{P})$.

4.2. First-order optimality conditions. In this subsection we derive the firstorder optimality system for the penalized-relaxed problems $\left(\mathrm{P}_{\gamma}\right)_{\gamma>0}$ using the mathematical programming approach in Banach spaces due to Zowe and Kurcyusz [42].

Let $\mathcal{X}$ and $\mathcal{Y}$ be real Banach spaces. For

$$
\begin{aligned}
F: \mathcal{X} \longrightarrow \mathbb{R} & \text { Fréchet-differentiable functional, } \\
g: \mathcal{X} \longrightarrow \mathcal{Y} & \text { continuously Fréchet-differentiable }
\end{aligned}
$$

we consider the following mathematical program:

$$
\min \{F(x) \mid g(x) \in M, x \in C\},
$$

where $C$ is a convex closed subset of $\mathcal{X}$, and $M$ is a closed cone in $\mathcal{Y}$ with vertex at 0 .

We suppose that problem (4.21) has an optimal solution $\hat{x}$, and we introduce the conical hulls of $C-\{\hat{x}\}$ and $M-\{y\}$, respectively, by

$$
\begin{aligned}
C(\hat{x}) & =\{x \in \mathcal{X} \mid \exists \beta \geq 0, \exists c \in C, x=\beta(c-\hat{x})\}, \\
M(y) & =\{z \in \mathcal{Y} \mid \exists \lambda \geq 0, \exists \zeta \in M, z=\zeta-\lambda y\} .
\end{aligned}
$$

The main result in [42] on the existence of a Lagrange multiplier for (4.21) is stated next. 
THEOREM 4.2. Let $\hat{x}$ be an optimal solution of problem (4.21) satisfying the constraints qualification

$$
g^{\prime}(\hat{x}) \cdot C(\hat{x})-M(g(\hat{x}))=\mathcal{Y} .
$$

Then there exists a Lagrange multiplier $\mu^{*} \in \mathcal{Y}^{*}$ such that

$$
\begin{aligned}
\left\langle\mu^{*}, z\right\rangle_{\mathcal{Y}^{*}, \mathcal{Y}} & \geq 0 \quad \forall z \in M, \\
\left\langle\mu^{*}, g(\hat{x})\right\rangle_{\mathcal{Y}^{*}, \mathcal{Y}} & =0 \\
F^{\prime}(\hat{x})-\mu^{*} \circ g^{\prime}(\hat{x}) & \in C(\hat{x})_{+},
\end{aligned}
$$

where $A_{+}=\left\{x^{*} \in \mathcal{X}^{*}:\left\langle x^{*}, a\right\rangle_{\mathcal{X}^{*}, \mathcal{X}} \geq 0\right.$ for all $\left.a \in A\right\}, \mathcal{Y}^{*}$ and $\mathcal{X}^{*}$ are the topological dual spaces of $\mathcal{Y}$ and $\mathcal{X}$, respectively, and $\left(\mu^{*} \circ g^{\prime}(\hat{x})\right) d=\left\langle\mu^{*}, g^{\prime}(\hat{x}) d\right\rangle_{\mathcal{Y}^{*}, \mathcal{Y}}$ for all $d \in$ $\mathcal{X}$.

We apply Theorem 4.2 to $\left(\mathrm{P}_{\gamma}\right)$. For this purpose we set

$$
\begin{aligned}
\mathcal{X} & =W(0, T) \times \mathcal{U} \times L^{2}(Q), \\
C & =W(0, T) \times U_{a d} \times L^{2}(Q), \\
\mathcal{Y} & =L^{2}\left(0, T, H^{-1}(\Omega)\right) \times L^{2}(\Omega) \times L^{2}(Q) \times \mathbb{R} \times \mathbb{R}, \\
\hat{x} & =\left(y_{\gamma}, u_{\gamma}, \xi_{\gamma}\right), \\
F(\hat{x}) & =J_{\gamma}\left(y_{\gamma}, u_{\gamma}\right), \\
M & =\{0\} \times\{0\} \times L^{2}(Q)_{+} \times \mathbb{R}^{+} \times \mathbb{R}^{+}, \\
g(\hat{x}) & =\left\{\begin{array}{l}
\frac{\partial y_{\gamma}}{\partial t}+\mathcal{A}\left(u_{\gamma}\right) y_{\gamma}-\xi_{\gamma}-f\left(u_{\gamma}\right), \\
y_{\gamma}(0), \\
\xi_{\gamma}, \\
\alpha_{\gamma}-\left(y_{\gamma}, \xi_{\gamma}\right)_{L^{2}(Q)}, \\
R-\frac{1}{2}\left\|\xi_{\gamma}\right\|_{L^{2}(Q)}^{2} .
\end{array}\right.
\end{aligned}
$$

The constraint qualification (4.22) in our setting requires the existence of $\left(c_{1}, c_{2}, c_{3}\right) \in$ $W(0, T) \times U_{a d} \times L^{2}(Q),\left(\zeta_{3}, \zeta_{4}, \zeta_{5}\right) \in L^{2}(Q)_{+} \times \mathbb{R}^{+} \times \mathbb{R}^{+}, \lambda \geq 0$, and $\beta \geq 0$ such that for an arbitrarily given $\left(y_{1}, y_{2}, y_{3}, y_{4}, y_{5}\right)$ in $L^{2}\left(0, T, H^{-1}(\Omega)\right) \times L^{2}(\Omega) \times L^{2}(Q) \times \mathbb{R} \times \mathbb{R}$ the following system holds:

$$
\begin{aligned}
& y_{1}=\frac{\partial c_{1}}{\partial t}+\mathcal{A}\left(u_{\gamma}\right) c_{1}+\mathcal{A}^{\prime}\left(\beta\left(c_{2}-u_{\gamma}\right)\right) y-c_{3}-f\left(\beta\left(c_{2}-u_{\gamma}\right)\right), \\
& y_{2}=c_{1}(0), \\
& y_{3}=c_{3}-\zeta_{3}+\lambda \xi_{\gamma}, \\
& y_{4}=-\left(y_{\gamma}, c_{3}\right)_{L^{2}(Q)}-\left(\xi_{\gamma}, c_{1}\right)_{L^{2}(Q)}-\zeta_{4}+\lambda\left(\alpha_{\gamma}-\left(y_{\gamma}, \xi_{\gamma}\right)_{L^{2}(Q)}\right), \\
& y_{5}=-\left(\xi_{\gamma}, c_{3}\right)_{L^{2}(Q)}-\zeta_{5}+\lambda\left(R-\frac{1}{2}\left\|\xi_{\gamma}\right\|_{L^{2}(Q)}^{2}\right) .
\end{aligned}
$$

By the classical theory of parabolic partial differential equations (see [17], for example), the system

$$
\begin{aligned}
\frac{\partial c_{1}}{\partial t}+\mathcal{A}\left(u_{\gamma}\right) c_{1} & =c_{3}+y_{1}, \\
c_{1}(0) & =y_{2}
\end{aligned}
$$

Copyright $($ C by SIAM. Unauthorized reproduction of this article is prohibited. 
admits a unique solution $c_{1} \in W(0, T)$ for every $y_{1} \in L^{2}\left(0, T, H^{-1}(\Omega)\right), y_{2} \in L^{2}(\Omega)$, and $c_{3} \in L^{2}(Q)$. Therefore, a solution of (4.26)-(4.30) is obtained by choosing

$$
\begin{aligned}
& c_{2} \in U_{a d}, \quad \beta=0 \\
& \lambda= \rho^{3}, \quad c_{3}=\rho c-\rho^{2} \xi_{\gamma}, \\
& c_{1} \text { solution of }(4.31)-(4.32), \\
& \zeta_{3}=\left(\rho^{3}-\rho^{2}\right) \xi_{\gamma}+\rho c-y_{3}, \\
& \zeta_{4}=\rho^{3}\left(\alpha_{\gamma}-\left(y_{\gamma}, \xi_{\gamma}\right)_{L^{2}(Q)}\right)+\rho^{2}\left(y_{\gamma}, \xi_{\gamma}\right)_{L^{2}(Q)}-\rho\left(y_{\gamma}, c\right)_{L^{2}(Q)} \\
& \quad-\left(\xi_{\gamma}, c_{1}\right)_{L^{2}(Q)}-y_{4}, \\
& \zeta_{5}=\rho^{3}\left(R-\frac{1}{2}\left\|\xi_{\gamma}\right\|_{L^{2}(Q)}^{2}\right)+\rho^{2}\left\|\xi_{\gamma}\right\|_{L^{2}(Q)}^{2}-\rho\left(\xi_{\gamma}, c\right)_{L^{2}(Q)}-y_{5}
\end{aligned}
$$

for some $c \in L^{2}(Q)$, with $c>0$ a.e. in $Q$, and $\rho>0$ large enough such that $\zeta_{3}, \zeta_{4}$, and $\zeta_{5}$ are nonnegative.

Consequently, problem $\left(\mathrm{P}_{\gamma}\right)$ satisfies the constraints qualification (4.22).

Proposition 4.3. Let $\left(y_{\gamma}, u_{\gamma}, \xi_{\gamma}\right)$ be a solution for problem $\left(\mathrm{P}_{\gamma}\right)$. Then there exists a Lagrange multiplier vector $\left(p_{\gamma}, \mu_{\gamma}, r_{\gamma}, \kappa_{\gamma}\right)$ in $W(0, T) \times L^{2}(Q) \times \mathbb{R} \times \mathbb{R}$ such that the following first-order optimality system holds:

$$
\begin{gathered}
\frac{\partial y_{\gamma}}{\partial t}+\mathcal{A}\left(u_{\gamma}\right) y_{\gamma}-f\left(u_{\gamma}\right)-\xi_{\gamma}=0, \\
y_{\gamma}(0)=0 \quad \text { a.e. in } \Omega, \\
-\frac{\partial p_{\gamma}}{\partial t}+\mathcal{A}^{*}\left(u_{\gamma}\right) p_{\gamma}=y_{\gamma}-y_{d}+r_{\gamma} \xi_{\gamma}-\max \left(0, \bar{\lambda}-\gamma y_{\gamma}\right), \\
p_{\gamma}(T)=y_{\gamma}(T)-y_{T} \quad \text { a.e. in } \Omega, \\
\nu\left\langle u_{\gamma}, u-u_{\gamma}\right\rangle_{\mathcal{U}}-\left\langle\mathcal{A}^{\prime}\left(u-u_{\gamma}\right) y_{\gamma}, p_{\gamma}\right\rangle+\left\langle f\left(u-u_{\gamma}\right), p_{\gamma}\right\rangle \geq 0 \quad \forall u \in U_{a d}, \\
\kappa_{\gamma} \xi_{\gamma}+p_{\gamma}-\mu_{\gamma}+r_{\gamma} y_{\gamma}=0 \quad \text { a.e. in } Q, \\
\xi_{\gamma} \geq 0 \text { a.e. in } Q, \quad \mu_{\gamma} \geq 0 \text { a.e. in } Q, \quad\left(\xi_{\gamma}, \mu_{\gamma}\right)_{L^{2}(Q)}=0, \\
\kappa_{\gamma} \geq 0, \quad \frac{1}{2}\left\|\xi_{\gamma}\right\|_{L^{2}(Q)}^{2} \leq R, \quad \frac{\kappa_{\gamma}}{2}\left\|\xi_{\gamma}\right\|_{L^{2}(Q)}^{2}=\kappa_{\gamma} R, \\
r_{\gamma} \geq 0, \quad\left(y_{\gamma}, \xi_{\gamma}\right)_{L^{2}(Q)} \leq \alpha_{\gamma}, r_{\gamma}\left(y_{\gamma}, \xi_{\gamma}\right)_{L^{2}(Q)}=r_{\gamma} \alpha_{\gamma} .
\end{gathered}
$$

5. First-order optimality conditions for limit problem (P). In this section we investigate the convergence of a sequence $\left(y_{\gamma}, u_{\gamma}, \xi_{\gamma}, p_{\gamma}, \mu_{\gamma}, r_{\gamma}, \kappa_{\gamma}\right)_{\gamma>0}$ satisfying the optimality conditions (4.33)-(4.41). For this purpose, we choose $\alpha_{\gamma}$ such that

$$
\frac{1}{\alpha_{\gamma} \sqrt{\gamma}} \leq C
$$

Moreover, we assume that

$$
\kappa_{\gamma} \gamma \leq C
$$

Here and in what follows, $C$ denotes a generic positive constant that may take different values at different occurrences but not depending on $\gamma$.

We recall that $\xi \in L^{2}(Q)$ in problem $(\mathrm{P})$. Hence, whenever the sequence $\left(\xi_{\gamma}\right)$ satisfies $\frac{\left\|\xi_{\gamma}\right\|_{L^{2}(Q)}^{2}}{2}<R$ for all sufficiently large $\gamma$, i.e., the second inequality constraint in (4.40) remains inactive for all sufficiently large $\gamma$, then $\kappa_{\gamma}=0$ and assumption (5.2) 
is satisfied. The latter behavior is the case when $\left(\xi_{\gamma}\right)$ converges to $\xi$ strongly in $L^{2}(Q)$ and it is observed, in particular, when we numerically solve the discrete problems for a large constant $R$.

We introduce the notation

$$
\begin{aligned}
\lambda_{\gamma} & =\max \left(0, \bar{\lambda}-\gamma y_{\gamma}\right), \\
\vartheta_{\gamma} & =r_{\gamma} \xi_{\gamma}-\lambda_{\gamma}, \\
N_{\gamma} & =\left\{(t, x) \in Q: y_{\gamma}(t, x)<0\right\}, \\
P_{\gamma} & =\left\{(t, x) \in Q: y_{\gamma}(t, x) \geq 0\right\}, \\
\Lambda_{\gamma} & =\left\{(t, x) \in Q: \bar{\lambda}(t, x)-\gamma y_{\gamma}(t, x) \geq 0\right\} .
\end{aligned}
$$

LEMma 5.1. Let $\left(y_{\gamma}, u_{\gamma}, \xi_{\gamma}, p_{\gamma}, \mu_{\gamma}, r_{\gamma}, \kappa_{\gamma}\right)$ be a solution of the optimality system (4.33)-(4.41). Then the following estimates hold:

(1)

$$
\left\|y_{\gamma}\right\|_{W(0, T)} \leq C, \quad\left\|y_{\gamma}(T)\right\|_{L^{2}(\Omega)} \leq C
$$

$$
\left(\xi_{\gamma}, p_{\gamma}\right)_{L^{2}(Q)} \leq 0
$$

$$
-\left(\lambda_{\gamma}, p_{\gamma}\right)_{L^{2}(Q)} \leq\|\bar{\lambda}\|_{L^{2}(Q)}\left\|p_{\gamma}\right\|_{L^{2}(Q)}+C
$$

(4)

$$
\left\|p_{\gamma}\right\|_{L^{2}\left(0, T, H_{0}^{1}(\Omega)\right)} \leq C, \quad\left\|p_{\gamma}(0)\right\|_{L^{2}(\Omega)} \leq C
$$

$$
\left\|u_{\gamma}\right\|_{\mathcal{U}} \leq C
$$

$$
r_{\gamma} \alpha_{\gamma} \leq C
$$

$$
\left|\left(\lambda_{\gamma}, y_{\gamma}\right)_{L^{2}(Q)}\right| \leq C,
$$

$$
\frac{1}{\sqrt{\gamma}}\left\|\lambda_{\gamma}\right\|_{L^{2}(Q)} \leq C \quad \forall \gamma \geq 1,
$$

(9)

$$
\left\|\frac{\partial p_{\gamma}}{\partial t}\right\|_{W(0, T)^{*}} \leq C
$$

$$
\left\|\vartheta_{\gamma}\right\|_{W(0, T)^{*}} \leq C .
$$

Copyright (c) by SIAM. Unauthorized reproduction of this article is prohibited. 
Proof. (1) By testing (4.33) against $y_{\gamma}$ and $\phi \in L^{2}\left(0, T, H_{0}^{1}(\Omega)\right)$, respectively, and using Lemma 2.1(iii), we obtain

$$
\begin{aligned}
\frac{1}{2}\left\|y_{\gamma}(T)\right\|_{L^{2}(\Omega)}^{2}+\omega\left\|y_{\gamma}\right\|_{L^{2}\left(0, T, H_{0}^{1}(\Omega)\right)}^{2} \leq & C_{f}\left\|y_{\gamma}\right\|_{L^{2}\left(0, T, H_{0}^{1}(\Omega)\right)} \\
& +\left\|\xi_{\gamma}\right\|_{L^{2}(Q)}\left\|y_{\gamma}\right\|_{L^{2}(Q)} \\
\left\langle\frac{\partial y_{\gamma}}{\partial t}, \phi\right\rangle \leq & C_{\mathcal{A}}\left\|y_{\gamma}\right\|_{L^{2}\left(0, T, H_{0}^{1}(\Omega)\right)}\|\phi\|_{L^{2}\left(0, T, H_{0}^{1}(\Omega)\right)} \\
& +C_{f}\|\phi\|_{L^{2}\left(0, T, H_{0}^{1}(\Omega)\right)}+\left\|\xi_{\gamma}\right\|_{L^{2}(Q)}\|\phi\|_{L^{2}(Q)} .
\end{aligned}
$$

Hence (1) follows from the boundedness of $\left\|\xi_{\gamma}\right\|_{L^{2}(Q)}$ in (4.40).

(2) Multiplying (4.38) by $\xi_{\gamma}$ and taking into account that $\left(\xi_{\gamma}, \mu_{\gamma}\right)_{L^{2}(Q)}=0$ from (4.39), we obtain

$$
\left(\xi_{\gamma}, p_{\gamma}\right)_{L^{2}(Q)}=-\kappa_{\gamma}\left\|\xi_{\gamma}\right\|_{L^{2}(Q)}^{2}-\alpha_{\gamma} r_{\gamma} \leq 0 .
$$

(3) From the definition of $\lambda_{\gamma}$ it follows that

$$
-\left(\lambda_{\gamma}, p_{\gamma}\right)_{L^{2}(Q)}=-\left(\bar{\lambda}, p_{\gamma}\right)_{\Lambda_{\gamma}}+\gamma\left(y_{\gamma}, p_{\gamma}\right)_{\Lambda_{\gamma} \cap P_{\gamma}}+\gamma\left(y_{\gamma}, p_{\gamma}\right)_{\Lambda_{\gamma} \cap N_{\gamma}=N_{\gamma}} .
$$

On the subset $\Lambda_{\gamma} \cap P_{\gamma}$ we have $0 \leq \gamma y_{\gamma} \leq \bar{\lambda}$. Then

$$
-\left(\lambda_{\gamma}, p_{\gamma}\right)_{L^{2}(Q)} \leq\|\bar{\lambda}\|_{L^{2}(Q)}\left\|p_{\gamma}\right\|_{L^{2}(Q)}+\gamma\left(y_{\gamma}, p_{\gamma}\right)_{N_{\gamma}} .
$$

Multiplying (4.38) by $\gamma y_{\gamma} \chi_{N_{\gamma}}$ with $\chi_{N_{\gamma}}$ the characteristic function of $N_{\gamma}$, we get

$$
\begin{aligned}
\gamma\left(y_{\gamma}, p_{\gamma}\right)_{N_{\gamma}} & =-\gamma \kappa_{\gamma}\left(y_{\gamma}, \xi_{\gamma}\right)_{N_{\gamma}}+\gamma\left(y_{\gamma}, \mu_{\gamma}\right)_{N_{\gamma}}-\gamma r_{\gamma}\left(y_{\gamma}, y_{\gamma}\right)_{N_{\gamma}}, \\
& \leq-\gamma \kappa_{\gamma}\left(y_{\gamma}, \xi_{\gamma}\right)_{N_{\gamma}} .
\end{aligned}
$$

Due to assumption (5.2) and the boundedness of $y_{\gamma}$ and $\xi_{\gamma}$ in $L^{2}(Q)$, we estimate

$$
-\gamma \kappa_{\gamma}\left(y_{\gamma}, \xi_{\gamma}\right)_{N_{\gamma}} \leq C
$$

Consequently,

$$
\gamma\left(y_{\gamma}, p_{\gamma}\right)_{N_{\gamma}} \leq C
$$

and, hence,

$$
-\left(\lambda_{\gamma}, p_{\gamma}\right)_{L^{2}(Q)} \leq\|\bar{\lambda}\|_{L^{2}(Q)}\left\|p_{\gamma}\right\|_{L^{2}(Q)}+C .
$$

(4) Using $p_{\gamma}$ as a test function in the adjoint equation (4.35) we obtain

$$
\begin{aligned}
\frac{1}{2}\left\|p_{\gamma}(0)\right\|_{L^{2}(\Omega)}^{2}+\left\langle\mathcal{A}\left(u_{\gamma}\right) p_{\gamma}, p_{\gamma}\right\rangle= & \left(y_{\gamma}-y_{d}, p_{\gamma}\right)_{L^{2}(Q)}+r_{\gamma}\left(\xi_{\gamma}, p_{\gamma}\right)_{L^{2}(Q)} \\
& -\left(\lambda_{\gamma}, p_{\gamma}\right)_{L^{2}(Q)}+\frac{1}{2}\left\|y_{\gamma}(T)-y_{T}\right\|_{L^{2}(Q)}^{2} .
\end{aligned}
$$

From (2.23), (1), (2), and (3) we see

$$
\frac{1}{2}\left\|p_{\gamma}(0)\right\|_{L^{2}(\Omega)}^{2}+\omega\left\|p_{\gamma}\right\|_{L^{2}\left(0, T, H_{0}^{1}(\Omega)\right)}^{2} \leq C\left\|p_{\gamma}\right\|_{L^{2}(Q)}+C .
$$

Copyright $@$ by SIAM. Unauthorized reproduction of this article is prohibited. 
In particular, we have

$$
\left\|p_{\gamma}\right\|_{L^{2}\left(0, T, H_{0}^{1}(\Omega)\right)} \leq C
$$

and

$$
\left\|p_{\gamma}(0)\right\|_{L^{2}(\Omega)} \leq C .
$$

(5) Let $u$ be an arbitrary element in $U_{a d}$. Then (4.37) yields

$$
\nu\left\|u-u_{\gamma}\right\|_{\mathcal{U}}^{2} \leq \nu\|u\|_{\mathcal{U}}\left\|u-u_{\gamma}\right\|_{\mathcal{U}}-\left\langle\mathcal{A}^{\prime}\left(u-u_{\gamma}\right) y_{\gamma}, p_{\gamma}\right\rangle+\left\langle f\left(u-u_{\gamma}\right), p_{\gamma}\right\rangle .
$$

From (2.20), (2.21), and the linearity of $f$ and $\mathcal{A}^{\prime}$ we have

$$
\left\|f\left(u-u_{\gamma}\right)\right\|_{L^{2}\left(0, T, H^{-1}(\Omega)\right)} \leq C
$$

and

$$
\left\|\mathcal{A}^{\prime}\left(u-u_{\gamma}\right) y\right\|_{L^{2}\left(0, T, H^{-1}(\Omega)\right)} \leq C\|y\|_{L^{2}\left(0, T, H_{0}^{1}(\Omega)\right)} .
$$

Hence

$$
\nu\left\|u-u_{\gamma}\right\|_{\mathcal{U}}^{2} \leq \nu\|u\|_{\mathcal{U}}\left\|u-u_{\gamma}\right\|_{\mathcal{U}}+C\left(\left\|y_{\gamma}\right\|_{L^{2}\left(0, T, H_{0}^{1}(\Omega)\right)}+1\right)\left\|p_{\gamma}\right\|_{L^{2}\left(0, T, H_{0}^{1}(\Omega)\right)} .
$$

Taking into account (1) and (4) we deduce that

$$
\left\|u-u_{\gamma}\right\|_{\mathcal{U}} \leq C
$$

and

$$
\left\|u_{\gamma}\right\|_{\mathcal{U}} \leq\left\|u-u_{\gamma}\right\|_{\mathcal{U}}+\|u\|_{\mathcal{U}} \leq C
$$

(6)-(7) Multiplying (4.38) by $\xi_{\gamma}$ and using (4.39) and (4.41), we get

$$
\begin{aligned}
r_{\gamma} \alpha_{\gamma} & =r_{\gamma}\left(y_{\gamma}, \xi_{\gamma}\right) \\
& =-\left(p_{\gamma}, \xi_{\gamma}\right)_{L^{2}(Q)}+\left(\mu_{\gamma}, \xi_{\gamma}\right)_{L^{2}(Q)}-\kappa_{\gamma}\left(\xi_{\gamma}, \xi_{\gamma}\right)_{L^{2}(Q)} \\
& =-\left(p_{\gamma}, \xi_{\gamma}\right)_{L^{2}(Q)}-\kappa_{\gamma}\left(\xi_{\gamma}, \xi_{\gamma}\right)_{L^{2}(Q)} \\
& \leq-\left(p_{\gamma}, \xi_{\gamma}\right)_{L^{2}(Q)} \\
& \leq\left\|p_{\gamma}\right\|_{L^{2}(Q)}\left\|\xi_{\gamma}\right\|_{L^{2}(Q)} .
\end{aligned}
$$

Then, from (4) and the boundedness of $\xi_{\gamma}$ in $L^{2}(Q)$ we deduce (6). By testing the adjoint equation (4.35) against $y_{\gamma}$, we obtain

$$
\left(\lambda_{\gamma}, y_{\gamma}\right)_{L^{2}(Q)}=\left\langle\frac{\partial p_{\gamma}}{\partial t}-\mathcal{A}^{*}\left(u_{\gamma}\right) p_{\gamma}, y_{\gamma}\right\rangle+\left(y_{\gamma}-y_{d}, y_{\gamma}\right)_{L^{2}(Q)}+r_{\gamma}\left(\xi_{\gamma}, y_{\gamma}\right)_{L^{2}(Q)} .
$$

On the other hand, from (4.33), (4.34), and (4.36) it follows that

$$
\begin{aligned}
& \left\langle\frac{\partial p_{\gamma}}{\partial t}-\mathcal{A}^{*}\left(u_{\gamma}\right) p_{\gamma}, y_{\gamma}\right\rangle \\
& \quad=\left\langle-\frac{\partial y_{\gamma}}{\partial t}-\mathcal{A}\left(u_{\gamma}\right) y_{\gamma}, p_{\gamma}\right\rangle+\frac{1}{2}\left(y_{\gamma}(T), p_{\gamma}(T)\right)_{L^{2}(\Omega)} \\
& \quad=-\left\langle f\left(u_{\gamma}\right) p_{\gamma}\right\rangle-\left(\xi_{\gamma}, p_{\gamma}\right)_{L^{2}(Q)}+\frac{1}{2}\left(y_{\gamma}(T), y_{\gamma}(T)-y_{T}\right)_{L^{2}(\Omega)}
\end{aligned}
$$

Copyright (c) by SIAM. Unauthorized reproduction of this article is prohibited. 
Thus

$$
\begin{aligned}
\left(\lambda_{\gamma}, y_{\gamma}\right)_{L^{2}(Q)}= & -\left\langle f\left(u_{\gamma}\right), p_{\gamma}\right\rangle-\left(\xi_{\gamma}, p_{\gamma}\right)_{L^{2}(Q)}+\frac{1}{2}\left(y_{\gamma}(T), y_{\gamma}(T)-y_{T}\right)_{L^{2}(\Omega)} \\
& +\left(y_{\gamma}-y_{d}, y_{\gamma}\right)_{L^{2}(Q)}+r_{\gamma}\left(\xi_{\gamma}, y_{\gamma}\right)_{L^{2}(Q)} .
\end{aligned}
$$

Therefore, from $(2.20),(1),(4),(6)$, and (5.4) we deduce that

$$
\left|\left(\lambda_{\gamma}, y_{\gamma}\right)_{L^{2}(Q)}\right| \leq C
$$

(8) The definition of $\lambda_{\gamma}$ yields

$$
\left\|\lambda_{\gamma}\right\|_{L^{2}(Q)}^{2}=\left(\lambda_{\gamma}, \lambda_{\gamma}\right)_{L^{2}(Q)}=\left(\lambda_{\gamma}, \bar{\lambda}\right)-\gamma\left(\lambda_{\gamma}, y_{\gamma}\right)_{\Lambda_{\gamma}} .
$$

Then

$$
\frac{1}{\gamma}\left\|\lambda_{\gamma}\right\|_{L^{2}(Q)}^{2} \leq \frac{1}{\gamma}\left\|\lambda_{\gamma}\right\|_{L^{2}(Q)}\|\bar{\lambda}\|_{L^{2}(Q)}+\left|\left(\lambda_{\gamma}, y_{\gamma}\right)_{L^{2}(Q)}\right|
$$

Using (7) we obtain

$$
\frac{1}{\gamma}\left\|\lambda_{\gamma}\right\|_{L^{2}(Q)}^{2} \leq \frac{1}{\sqrt{\gamma}}\left\|\lambda_{\gamma}\right\|_{L^{2}(Q)}\|\bar{\lambda}\|_{L^{2}(Q)}+C \quad \forall \gamma \geq 1 .
$$

In particular we infer

$$
\frac{1}{\sqrt{\gamma}}\left\|\lambda_{\gamma}\right\|_{L^{2}(Q)} \leq C \quad \forall \gamma \geq 1
$$

(9)-(10) Let $v \in W(0, T)$. Using integration by parts, we obtain

$$
\left\langle\frac{\partial p_{\gamma}}{\partial t}, v\right\rangle=-\left\langle\frac{\partial v}{\partial t}, p_{\gamma}\right\rangle+\left(y_{\gamma}(T)-y_{T}, v(T)\right)_{L^{2}(\Omega)}-\left(p_{\gamma}(0), v(0)\right)_{L^{2}(\Omega)} .
$$

The continuous injection of $W(0, T)$ into $\mathcal{C}^{0}\left(0, T, L^{2}(\Omega)\right)$ yields

$$
\left\langle\frac{\partial p_{\gamma}}{\partial t}, v\right\rangle \leq\left(\left\|p_{\gamma}\right\|_{L^{2}\left(0, T, H_{0}^{1}(\Omega)\right)}+\left\|y_{\gamma}(T)-y_{T}\right\|_{L^{2}(\Omega)}+\left\|p_{\gamma}(0)\right\|_{L^{2}(\Omega)}\right)\|v\|_{W(0, T)} .
$$

From (1) and (4) we deduce that

$$
\left\|\frac{\partial p_{\gamma}}{\partial t}\right\|_{W(0, T)^{*}} \leq C
$$

The boundedness of $\vartheta_{\gamma}=r_{\gamma} \xi_{\gamma}-\lambda_{\gamma}$ in $W(0, T)^{*}$ follows from the adjoint equation, (5.6) and Lemma 2.1(iii).

Now we can state the main result of this section.

ThEOREM 5.2. Let $\left(y_{\gamma}, u_{\gamma}, \xi_{\gamma}, p_{\gamma}, \mu_{\gamma}, r_{\gamma}, \kappa_{\gamma}\right)_{\gamma>0}$ be a sequence of solutions of the optimality system (4.33)-(4.41). Then there exists $\left(y^{\star}, u^{\star}, \xi^{\star}, p^{\star}, \vartheta^{\star}\right) \in W(0, T) \times$ $U_{a d} \times L^{2}(\Omega) \times L^{2}\left(0, T, H_{0}^{1}(\Omega)\right) \times W(0, T)^{*}$ and a subsequence still denoted by

Copyright (c) by SIAM. Unauthorized reproduction of this article is prohibited. 
$\left(y_{\gamma}, u_{\gamma}, \xi_{\gamma}, p_{\gamma}, \mu_{\gamma}, r_{\gamma}, \kappa_{\gamma}\right)_{\gamma>0}$ such that

$$
\begin{array}{cl}
u_{\gamma} \rightarrow u^{\star} & \text { in } \mathcal{U}, \\
\xi_{\gamma} \rightarrow \xi^{\star} & \text { in } L^{2}(Q), \\
y_{\gamma} \rightarrow y^{\star} & \text { in } W(0, T), \\
u_{\gamma} \longrightarrow u^{\star} & \text { in } L^{\infty}\left(0, T, W^{1, \infty}(\Omega)\right), \\
y_{\gamma} \longrightarrow y^{\star} & \text { in } L^{2}\left(0, T, H_{0}^{1}(\Omega)\right), \\
y_{\gamma}(T) \longrightarrow y^{\star}(T) & \text { in } L^{2}(\Omega), \\
p_{\gamma} \rightarrow p^{\star} & \text { in } L^{2}\left(0, T, H_{0}^{1}(\Omega)\right), \\
\vartheta_{\gamma} \stackrel{*}{\rightarrow} \vartheta^{\star} & \text { in } W(0, T)^{*} .
\end{array}
$$

The limit element $\left(y^{\star}, u^{\star}, \xi^{\star}, p^{\star}, \vartheta^{\star}\right)$ satisfies the following optimality system:

$$
\begin{gathered}
\frac{\partial y^{\star}}{\partial t}+\mathcal{A}\left(u^{\star}\right) y^{\star}-f\left(u^{\star}\right)-\xi^{\star}=0 \quad \text { in } L^{2}\left(0, T, H^{-1}(\Omega)\right), \\
y^{\star}(0)=0 \quad \text { a.e. in } \Omega, \\
\left\langle\left(\frac{\partial}{\partial t}+\mathcal{A}\left(u^{\star}\right)\right) \varphi, p^{\star}\right\rangle=\left\langle y^{\star}-y_{d}, \varphi\right\rangle+\left\langle y^{\star}(T)-y_{T}, \varphi(T)\right\rangle+\left\langle\vartheta^{\star}, \varphi\right\rangle \\
\forall \varphi \in Z=\{z \in W(0, T), z(0)=0\}, \\
\nu\left(u^{\star}, u-u^{\star}\right)_{\mathcal{U}}-\left\langle\mathcal{A}^{\prime}\left(u-u^{\star}\right) y^{\star}, p^{\star}\right\rangle+\left\langle f\left(u-u^{\star}\right), p^{\star}\right\rangle \geq 0 \quad \forall u \in U_{a d}, \\
y^{\star} \geq 0 \text { a.e. in } Q, \quad \xi^{\star} \geq 0 \text { a.e.in } Q, \quad\left(\xi^{\star}, y^{\star}\right)_{L^{2}(Q)}=0, \\
R \geq \frac{1}{2}\left\|\xi^{\star}\right\|_{L^{2}(\Omega)}^{2}, \\
\lim _{\gamma \longrightarrow 0}\left(p_{\gamma}, \xi_{\gamma}\right)_{L^{2}(Q)}=0, \\
\lim _{\gamma \longrightarrow \infty}\left(\vartheta_{\gamma}, y_{\gamma}^{+}\right)_{L^{2}(Q)}=0, \\
\lim _{\gamma \longrightarrow \infty}\left(\vartheta_{\gamma}, p_{\gamma}\right)_{L^{2}(Q)} \leq 0,
\end{gathered}
$$

with $y_{\gamma}^{+}=\max (0, y)$.

Proof. The assertions (5.7)-(5.17) are consequences of (4.33)-(4.36), Proposition 3.2, the compactness results given in Lemma 3.1, and the estimates in (1), (4), (5), (9), and (10) established in Lemma 5.1. To show (5.19)-(5.20) we proceed as in the proof of Theorem 4.1 (see (4.8)-(4.19)). Furthermore, from (5.10), (5.11), (5.13), and Lemma 2.1(ii) it follows that for every $u \in U_{a d}$,

$$
\begin{aligned}
\lim _{\gamma \longrightarrow \infty}\left\langle\mathcal{A}^{\prime}\left(u-u_{\gamma}\right) y_{\gamma}, p_{\gamma}\right\rangle & =\left\langle\mathcal{A}^{\prime}\left(u-u^{\star}\right) y^{\star}, p^{\star}\right\rangle, \\
\lim _{\gamma \longrightarrow \infty}\left\langle f\left(u-u_{\gamma}\right), p_{\gamma}\right\rangle & =\left\langle f\left(u-u^{\star}\right), p^{\star}\right\rangle .
\end{aligned}
$$

Moreover, the weak lower semicontinuity of the norm in $\mathcal{U}$ yields

$$
\liminf _{\gamma \longrightarrow \infty} \nu\left(u_{\gamma}, u-u_{\gamma}\right)_{\mathcal{U}} \leq \nu\left(u^{\star}, u-u^{\star}\right)_{\mathcal{U}} .
$$

Copyright (c) by SIAM. Unauthorized reproduction of this article is prohibited. 
Hence, letting $\gamma$ tend to $\infty$ in (4.37) and taking into account (5.24)-(5.26), we get (5.18).

Next we prove $\lim _{\gamma \longrightarrow \infty}\left(p_{\gamma}, \xi_{\gamma}\right)=0$. If $\left(r_{\gamma}\right)$ is bounded, then $\lim _{\gamma} \longrightarrow \infty\left(p_{\gamma}, \xi_{\gamma}\right)=$ 0 follows immediately from (5.5), (5.2), (5.1), and the boundness of $\xi_{\gamma}$ in $L^{2}(Q)$. In the case where $\left(r_{\gamma}\right)$ is unbounded, we take $p_{\gamma}$ as a test function in the adjoint system (4.35)-(4.36) and estimate

$$
\begin{aligned}
r_{\gamma}\left(\xi_{\gamma}, p_{\gamma}\right)_{L^{2}(Q)} \geq & \omega\left\|p_{\gamma}\right\|_{L^{2}\left(0, T, H_{0}^{1}(\Omega)\right)}^{2}+\frac{1}{2}\left\|p_{\gamma}(0)\right\|_{L^{2}(\Omega)}^{2}-\frac{1}{2}\left\|y_{\gamma}(T)-y_{T}\right\|_{L^{2}(Q)}^{2} \\
& +\left(\lambda_{\gamma}, p_{\gamma}\right)_{L^{2}(Q)}+\left(y_{d}-y_{\gamma}, p_{\gamma}\right)_{L^{2}(Q)} .
\end{aligned}
$$

This, together with (1), (3), (4), and (7) of Lemma 5.1, yields

$$
r_{\gamma}\left(\xi_{\gamma}, p_{\gamma}\right)_{L^{2}(Q)} \geq-C
$$

which implies

$$
\lim _{\gamma \longrightarrow \infty}\left(\xi_{\gamma}, p_{\gamma}\right)_{L^{2}(Q)} \geq 0
$$

From (2) of Lemma 5.1 we have

$$
\lim _{\gamma \longrightarrow \infty}\left(p_{\gamma}, \xi_{\gamma}\right)_{L^{2}(Q)} \leq 0,
$$

and as a consequence,

$$
\lim _{\gamma \longrightarrow \infty}\left(p_{\gamma}, \xi_{\gamma}\right)_{L^{2}(Q)}=0
$$

In addition, we find

$$
\lim _{\gamma \longrightarrow \infty} r_{\gamma} \alpha_{\gamma}=0
$$

due to (5.5).

Moreover, we have

$$
0 \leq\left(\lambda_{\gamma}, y_{\gamma}^{+}\right)_{L^{2}(Q)} \leq\left(\bar{\lambda}, y_{\gamma}\right)_{\Lambda_{\gamma} \cap P_{\gamma}}-\gamma\left(y_{\gamma}, y_{\gamma}\right)_{\Lambda_{\gamma} \cap P_{\gamma}} \leq \frac{1}{\gamma}\|\bar{\lambda}\|_{L^{2}(Q)}^{2}
$$

and

$$
\begin{aligned}
r_{\gamma}\left(\xi_{\gamma}, y_{\gamma}^{+}\right)_{L^{2}(Q)} & =r_{\gamma}\left(\xi_{\gamma}, y_{\gamma}\right)_{L^{2}(Q)}-r_{\gamma}\left(\xi_{\gamma}, y_{\gamma}\right)_{L^{2}\left(N_{\gamma}\right)} \\
& =r_{\gamma} \alpha_{\gamma}-r_{\gamma}\left(\xi_{\gamma}, y_{\gamma}\right)_{L^{2}\left(N_{\gamma}\right)} \\
& \leq r_{\gamma} \alpha_{\gamma}+r_{\gamma} \alpha_{\gamma} \frac{1}{\alpha_{\gamma} \sqrt{\gamma}}\left\|\xi_{\gamma}\right\|_{L^{2}(Q)}\left\|\sqrt{\gamma} y_{\gamma}\right\|_{L^{2}\left(N_{\gamma}\right)} \\
& \leq C r_{\gamma} \alpha_{\gamma}\left\|\sqrt{\gamma} y_{\gamma}\right\|_{L^{2}\left(N_{\gamma}\right)} .
\end{aligned}
$$

The last inequality is due to the boundedness of $\left\|\xi_{\gamma}\right\|$ in $L^{2}(Q)$ and (5.1). From (5.28) and (5.29) it follows that

$$
\begin{aligned}
\left(\vartheta_{\gamma}, y_{\gamma}^{+}\right)_{L^{2}(Q)} & =\left(r_{\gamma} \xi_{\gamma}-\lambda_{\gamma}, y_{\gamma}^{+}\right)_{L^{2}(Q)} \\
& \leq C r_{\gamma} \alpha_{\gamma}\left\|\sqrt{\gamma} y_{\gamma}\right\|_{L^{2}\left(N_{\gamma}\right)}+\frac{1}{\gamma}\|\bar{\lambda}\|_{L^{2}(Q)}^{2} .
\end{aligned}
$$

Copyright (c) by SIAM. Unauthorized reproduction of this article is prohibited. 
Hence, due to (5.27), it suffices to show that $\left\|\sqrt{\gamma} y_{\gamma}\right\|_{L^{2}\left(N_{\gamma}\right)}$ is bounded for proving (5.22). For this purpose we use (7) of Lemma 5.1 and find

$$
\left(\lambda_{\gamma}, y_{\gamma}\right)_{L^{2}(Q)}=\left(\bar{\lambda}-\gamma y_{\gamma}, y_{\gamma}\right)_{L^{2}\left(\Lambda_{\gamma}\right)} \geq-C
$$

and further

$$
\left(\bar{\lambda}, y_{\gamma}\right)_{L^{2}\left(\Lambda_{\gamma}\right)}-\gamma\left(y_{\gamma}, y_{\gamma}\right)_{L^{2}\left(\Lambda_{\gamma} \cap P_{\gamma}\right)}-\gamma\left(y_{\gamma}, y_{\gamma}\right)_{L^{2}\left(N_{\gamma}\right)} \geq-C
$$

as well as

$$
\left(\bar{\lambda}, y_{\gamma}\right)_{L^{2}\left(\Lambda_{\gamma}\right)}+C \geq \gamma\left(y_{\gamma}, y_{\gamma}\right)_{L^{2}\left(N_{\gamma}\right)} .
$$

Note that due to (1) of Lemma 5.1 we have

$$
\left(\bar{\lambda}, y_{\gamma}\right)_{L^{2}\left(\Lambda_{\gamma}\right)} \leq\|\bar{\lambda}\|_{L^{2}(\Omega)}\left\|y_{\gamma}\right\|_{L^{2}(\Omega)} \leq C .
$$

Consequently,

$$
\left\|\sqrt{\gamma} y_{\gamma}\right\|_{L^{2}\left(N_{\gamma}\right)} \leq C
$$

and (5.22) holds true.

Finally, we prove (5.23). We multiply (4.38) by $\lambda_{\gamma}$ and estimate

$$
\begin{aligned}
-\left(p_{\gamma}, \lambda_{\gamma}\right)_{L^{2}(Q)}= & \kappa_{\gamma}\left(\xi_{\gamma}, \lambda_{\gamma}\right)_{L^{2}(Q)}-\left(\mu_{\gamma}, \lambda_{\gamma}\right)_{L^{2}(Q)}+r_{\gamma}\left(y_{\gamma}, \lambda_{\gamma}\right)_{L^{2}(Q)} \\
\leq & \kappa_{\gamma}\left(\xi_{\gamma}, \lambda_{\gamma}\right)_{L^{2}(Q)}+r_{\gamma}\left(y_{\gamma}, \lambda_{\gamma}\right)_{L^{2}(Q)} \\
\leq & \kappa_{\gamma}\left(\xi_{\gamma}, \bar{\lambda}\right)_{L^{2}\left(\Lambda_{\gamma}\right)}-\kappa_{\gamma} \gamma\left(\xi_{\gamma}, y_{\gamma}\right)_{L^{2}\left(N_{\gamma}\right)} \\
& +r_{\gamma}\left(y_{\gamma}, \bar{\lambda}\right)_{L^{2}\left(\Lambda_{\gamma} \cap P_{\gamma}\right)}-\gamma r_{\gamma}\left(y_{\gamma}, y_{\gamma}\right)_{L^{2}\left(\Lambda_{\gamma} \cap P_{\gamma}\right)} .
\end{aligned}
$$

Since $\frac{\bar{\lambda}}{\gamma} \geq y_{\gamma} \geq 0$ on $\Lambda_{\gamma} \cap P_{\gamma}$, we obtain

$$
-\left(p_{\gamma}, \lambda_{\gamma}\right)_{L^{2}(Q)} \leq \kappa_{\gamma}\left(\xi_{\gamma}, \bar{\lambda}\right)_{L^{2}\left(\Lambda_{\gamma}\right)}-\kappa_{\gamma} \sqrt{\gamma}\left(\xi_{\gamma}, \sqrt{\gamma} y_{\gamma}\right)_{L^{2}\left(N_{\gamma}\right)}+\frac{r_{\gamma}}{\gamma}(\bar{\lambda}, \bar{\lambda})_{L^{2}\left(\Lambda_{\gamma} \cap P_{\gamma}\right)} .
$$

From (5.1), assumption (5.2), and (5.27), we infer

$$
\lim _{\gamma \longrightarrow \infty} \kappa_{\gamma} \sqrt{\gamma}=0 \text { and } \lim _{\gamma \longrightarrow \infty} \frac{r_{\gamma}}{\gamma}=\lim _{\gamma \longrightarrow \infty} \frac{1}{\sqrt{\gamma}} \frac{r_{\gamma} \alpha_{\gamma}}{\sqrt{\gamma} \alpha_{\gamma}}=0 .
$$

Hence, using the boundedness of $\xi_{\gamma}$ in $L^{2}(Q)$ and (5.32), we get

$$
\limsup _{\gamma \longrightarrow \infty}-\left(p_{\gamma}, \lambda_{\gamma}\right)_{L^{2}(Q)} \leq 0 .
$$

On the other hand, by (2) of Lemma 5.1 we have

$$
\left(p_{\gamma}, \vartheta_{\gamma}\right)=r_{\gamma}\left(p_{\gamma}, \xi_{\gamma}\right)-\left(p_{\gamma}, \lambda_{\gamma}\right) \leq-\left(p_{\gamma}, \lambda_{\gamma}\right) .
$$

Therefore, from (5.33) and (5.34), we deduce

$$
\limsup _{\gamma \longrightarrow \infty}\left(p_{\gamma}, \vartheta_{\gamma}\right) \leq 0,
$$

which completes the proof of the theorem.

Copyright (c) by SIAM. Unauthorized reproduction of this article is prohibited. 
6. An active-set-Newton solver for the discrete optimality system. In this section we introduce a Newton-type algorithm for solving the discretized counterpart of the first-order optimality system of Theorem 5.2. We assume that after an appropriate discretization in space and time (for details, we refer to section 7 ) the discrete system reads

$$
\begin{aligned}
R_{S}\left(\boldsymbol{y}^{\star}, \boldsymbol{u}^{\star}\right)-\boldsymbol{\xi}^{\star} & =0, \\
\boldsymbol{y}^{\star} \geq 0, \quad \boldsymbol{\xi}^{\star} \geq 0, \quad \boldsymbol{y}^{\star} \odot \boldsymbol{\xi}^{\star} & =0, \\
R_{A}\left(\boldsymbol{y}^{\star}, \boldsymbol{p}^{\star}, \boldsymbol{u}^{\star}\right)-\boldsymbol{\vartheta}^{\star} & =0, \\
R_{C}\left(\boldsymbol{y}^{\star}, \boldsymbol{p}^{\star}, \boldsymbol{u}^{\star}\right) \cdot\left(\boldsymbol{u}-\boldsymbol{u}^{\star}\right) & \geq 0 \quad \forall \boldsymbol{u} \in U_{a d}^{h}, \\
\boldsymbol{y}^{\star} \odot \boldsymbol{\vartheta}^{\star} & =0, \\
\boldsymbol{p}^{\star} \odot \boldsymbol{\xi}^{\star} & =0, \\
\boldsymbol{p}^{\star} \odot \boldsymbol{\vartheta}^{\star} & \leq 0 .
\end{aligned}
$$

By $R_{S}: \mathbb{R}^{N_{y}} \times \mathbb{R}^{N_{u}} \rightarrow \mathbb{R}^{N_{y}}, R_{A}: \mathbb{R}^{N_{y}} \times \mathbb{R}^{N_{y}} \times \mathbb{R}^{N_{u}} \rightarrow \mathbb{R}^{N_{y}}$, and $R_{C}: \mathbb{R}^{N_{y}} \times$ $\mathbb{R}^{N_{y}} \times \mathbb{R}^{N_{u}} \rightarrow \mathbb{R}^{N_{u}}$ we denote $\mathcal{C}^{1}$-functions corresponding to the discrete state and adjoint system as well as the discrete variational inequality (5.18). For a particular realization of these quantities and further details in the context of option pricing, we refer to [5]. By $\odot$ we denote the componentwise multiplication of two vectors. The discrete admissible set $U_{a d}^{h}$ is given by

$$
U_{a d}^{h}:=\left\{\boldsymbol{u} \in \mathbb{R}^{N_{u}}: 0<\underline{\boldsymbol{u}} \leq \boldsymbol{u} \leq \overline{\boldsymbol{u}}\right\}
$$

with $\underline{\boldsymbol{u}}, \overline{\boldsymbol{u}} \in \mathbb{R}^{N_{u}}$. We assume here that the constraints

$$
R \geq \frac{1}{2}\|\xi\|_{L^{2}(\Omega)}^{2} \quad \text { and } \quad\left|\frac{\partial u(t, x)}{\partial x}\right| \leq M \text { in } U_{a d}
$$

are fulfilled automatically due to discretization.

Concerning the solution algorithm for (6.1)-(6.6), we adapt a method proposed in [21] for inverse coefficient problems in elliptic variational inequalities. For the subsequent presentation we assume that $\boldsymbol{\xi}^{\star}$ and $\boldsymbol{y}^{\star}$ satisfy a strict complementarity condition, i.e.,

$$
\mathcal{A S}\left(\boldsymbol{y}^{\star}\right)=\mathcal{I S}\left(\boldsymbol{\xi}^{\star}\right)
$$

with the active and inactive sets

$$
\begin{aligned}
& \mathcal{A S}\left(\boldsymbol{y}^{\star}\right)=\left\{i \in\left\{1 \cdots N_{y}\right\}: \boldsymbol{y}_{i}^{\star}=0\right\}, \\
& \mathcal{I S}\left(\boldsymbol{y}^{\star}\right)=\left\{i \in\left\{1 \cdots N_{y}\right\}: \boldsymbol{y}_{i}^{\star}>0\right\},
\end{aligned}
$$

respectively, and similarly for $\mathcal{A S}\left(\boldsymbol{\xi}^{\star}\right)$ and $\mathcal{I S}\left(\boldsymbol{\xi}^{\star}\right)$. This assumption implies

$$
\begin{aligned}
R_{S}\left(\boldsymbol{y}^{\star}, \boldsymbol{u}^{\star}\right)_{i}=0, & i \in \mathcal{I S}\left(\boldsymbol{y}^{\star}\right), \\
\boldsymbol{y}_{i}^{\star}=0, & i \in \mathcal{A S}\left(\boldsymbol{y}^{\star}\right), \\
R_{A}\left(\boldsymbol{y}^{\star}, \boldsymbol{p}^{\star}, \boldsymbol{u}^{\star}\right)_{i}=0, & i \in \mathcal{I S}\left(\boldsymbol{y}^{\star}\right), \\
\boldsymbol{p}_{i}^{\star}=0, & i \in \mathcal{A S}\left(\boldsymbol{y}^{\star}\right) .
\end{aligned}
$$

In order to guarantee the positive definiteness of the discretization $A^{h}(\boldsymbol{u})$ of the operator $\mathcal{A}(u)$, an interior point treatment with respect to $\underline{\boldsymbol{u}} \leq \boldsymbol{u} \leq \overline{\boldsymbol{u}}$ is proposed; 
see [39] for a general account of primal-dual path-following interior point methods. With respect to the positive definiteness of $A^{h}(\boldsymbol{u})$, in particular the lower bound on $\boldsymbol{u}$ is important. For this purpose we replace the variational inequality (6.4) by

$$
\begin{aligned}
R_{C}(\boldsymbol{y}, \boldsymbol{p}, \boldsymbol{u})-\boldsymbol{q}_{\boldsymbol{l}}+\boldsymbol{q}_{\boldsymbol{u}} & =0, \\
\boldsymbol{q}_{\boldsymbol{l}} \odot(\boldsymbol{u}-\underline{\boldsymbol{u}}) & =\varkappa \mathbb{1}, \\
\boldsymbol{q}_{\boldsymbol{u}} \odot(\overline{\boldsymbol{u}}-\boldsymbol{u}) & =\varkappa \mathbb{1},
\end{aligned}
$$

where $\mathbb{1}:=(1, \ldots, 1)$, and $\boldsymbol{q}_{\boldsymbol{l}}, \boldsymbol{q}_{\boldsymbol{u}} \in \mathbb{R}_{+}^{N_{u}}$ represent approximate Lagrange multipliers (dual variables) for the lower and upper bound constraint on $\boldsymbol{u}$, respectively. Moreover, $\varkappa$ denotes a positive parameter which tends to zero in the iterative framework outlined below for solving the overall nonlinear system. Conceptually it chooses some initial approximation $\boldsymbol{u}^{(0)}$ of the primal variable $\boldsymbol{u}^{\star}$ with $\underline{\boldsymbol{u}}<\boldsymbol{u}^{(0)}<\overline{\boldsymbol{u}}$ and $\boldsymbol{q}_{l}^{(\mathbf{0})}, \boldsymbol{q}_{\boldsymbol{u}}^{(\mathbf{0})}>0$ along with some $\varkappa^{(0)}>0$. Then, based on the solution of the linearization of (6.8)-(6.11) at a current iterate $\left(\boldsymbol{y}^{(\boldsymbol{k})}, \boldsymbol{u}^{(\boldsymbol{k})}, \boldsymbol{p}^{(\boldsymbol{k})}\right)$ and (6.12)-(6.14) at $\left(\boldsymbol{y}^{(k)}, \boldsymbol{u}^{(k)}, \boldsymbol{p}^{(k)}, \boldsymbol{q}_{\boldsymbol{u}}^{(\boldsymbol{k})}, \boldsymbol{q}_{l}^{(k)}\right)$, a new iterate is generated with $\underline{\boldsymbol{u}}<\boldsymbol{u}^{(k+1)}<\overline{\boldsymbol{u}}$, $\boldsymbol{q}_{\boldsymbol{l}}^{(\boldsymbol{k + 1})}, \boldsymbol{q}_{\boldsymbol{u}}^{(\boldsymbol{k}+\mathbf{1})}>0$, and $0<\varkappa^{(k+1)}<\varkappa^{(k)}$ is chosen. Hence, $\left(\boldsymbol{u}^{(\boldsymbol{k})}\right)$ always stays in the interior of $\left\{\boldsymbol{u} \in \boldsymbol{R}^{N_{u}}: \underline{\boldsymbol{u}} \leq \boldsymbol{u} \leq \overline{\boldsymbol{u}}\right\}$, which guarantees the positive definiteness of $A^{h}\left(\boldsymbol{u}^{(k)}\right)$ for all $k$. As $\varkappa^{(k)}$ tends to zero, $\boldsymbol{u}^{(k)}$ converges to the solution and, thus, may approach the bounds. For an alternative motivation of (6.12)-(6.14) related to a barrier problem formulation of the parameter identification problem, we refer to [21].

Next we define our algorithm. Below, for ease of notation, we use $R_{S}^{(k)}:=$ $R_{S}\left(\boldsymbol{y}^{(\boldsymbol{k})}, \boldsymbol{u}^{(\boldsymbol{k})}\right)$ and similarly for $R_{A}^{(k)}$ and $R_{C}^{(k)}$.

\subsection{Algorithm.}

Fix the parameters $\epsilon \in(0,1), \zeta \in(0,1)$, and $k_{\max } \in \mathbb{N}$.

(1) Choose $\boldsymbol{p}^{(0)}, \boldsymbol{u}^{(0)}, \boldsymbol{q}_{l}^{(0)}$, and $\boldsymbol{q}_{\boldsymbol{u}}^{(0)}$ such that

$$
\underline{\boldsymbol{u}}_{i}<\boldsymbol{u}_{i}^{(\mathbf{0})}<\overline{\boldsymbol{u}}_{i}, \quad \boldsymbol{q}_{\boldsymbol{l}, \boldsymbol{i}}^{(\mathbf{0})}>0, \quad \boldsymbol{q}_{\boldsymbol{u}, \boldsymbol{i}}^{(\mathbf{0})}>0 \quad \forall i \in\left\{1 \cdots N_{u}\right\} .
$$

Set $k:=0$. Define

$$
\varkappa^{(0)}=\max \left(\left(\boldsymbol{u}^{(0)}-\underline{\boldsymbol{u}}^{(0)}\right)^{\top} \boldsymbol{q}_{\boldsymbol{l}}^{(0)},\left(\overline{\boldsymbol{u}}^{(0)}-\boldsymbol{u}^{(0)}\right)^{\top} \boldsymbol{q}_{\boldsymbol{u}}^{(0)}\right) .
$$

(2) Unless $k>k_{\max }$ or some stopping criterion is satisfied, perform the following steps:

(a) Find $\left(\boldsymbol{y}^{(k)}, \boldsymbol{\xi}^{(k)}\right)$ such that

$$
\begin{gathered}
R_{S}\left(\boldsymbol{y}^{(k)}, \boldsymbol{u}^{(k)}\right)-\boldsymbol{\xi}^{(k)}=0 \\
\boldsymbol{y}^{(k)} \geq 0, \quad \boldsymbol{\xi}^{(k)} \geq 0, \quad \boldsymbol{y}^{(\boldsymbol{k})} \odot \boldsymbol{\xi}^{(\boldsymbol{k})}=0 .
\end{gathered}
$$

(b) Determine the estimates of the active and the inactive set

$$
\mathcal{A S}\left(\boldsymbol{y}^{(k)}\right)=\left\{i: \boldsymbol{y}_{i}^{(k)}=0\right\}, \quad \mathcal{I S}\left(\boldsymbol{y}^{(k)}\right)=\left\{i: \boldsymbol{y}_{i}^{(k)}>0\right\},
$$

respectively.

Copyright $@$ by SIAM. Unauthorized reproduction of this article is prohibited. 
(c) Compute the update direction $\left(\boldsymbol{d} \boldsymbol{y}^{(\boldsymbol{k})}, \boldsymbol{d} \boldsymbol{p}^{(\boldsymbol{k})}, \boldsymbol{d} \boldsymbol{u}^{(\boldsymbol{k})}, \boldsymbol{d} \boldsymbol{q}_{l}^{(k)}, \boldsymbol{d} \boldsymbol{q}_{u}^{(k)}\right)$ by solving the linear system

$$
\begin{aligned}
& \left(\frac{\partial R_{S}^{(k)}}{\partial \boldsymbol{y}} \boldsymbol{d} \boldsymbol{y}^{(\boldsymbol{k})}+\frac{\partial R_{S}^{(k)}}{\partial \boldsymbol{u}} \boldsymbol{d} \boldsymbol{u}^{(\boldsymbol{k})}\right)_{i \in \mathcal{I S}\left(\boldsymbol{y}^{(k)}\right)}=-\left(R_{S}^{(k)}\right)_{i \in \mathcal{I} \mathcal{S}\left(\boldsymbol{y}^{(k)}\right)} \\
& \left(\boldsymbol{d} \boldsymbol{y}^{(k)}\right)_{i \in \mathcal{A S}\left(\boldsymbol{y}^{(k)}\right)}=0, \\
& \left(\frac{\partial R_{A}^{(k)}}{\partial \boldsymbol{y}} \boldsymbol{d} \boldsymbol{y}^{(\boldsymbol{k})}+\frac{\partial R_{A}^{(k)}}{\partial \boldsymbol{p}} \boldsymbol{d} \boldsymbol{p}^{(\boldsymbol{k})}+\frac{\partial R_{A}^{(k)}}{\partial \boldsymbol{u}} \boldsymbol{d} \boldsymbol{u}^{(\boldsymbol{k})}\right)_{i \in \mathcal{I} \mathcal{S}\left(\boldsymbol{y}^{(k)}\right)} \\
& =-\left(R_{A}^{(k)}\right)_{i \in \mathcal{I S}\left(\boldsymbol{y}^{(k)}\right)}, \\
& \left(\boldsymbol{d} \boldsymbol{p}^{(k)}\right)_{i \in \mathcal{A S}\left(\boldsymbol{y}^{(k)}\right)}=-\left(\boldsymbol{p}^{(k)}\right)_{i \in \mathcal{A S}\left(\boldsymbol{y}^{(k)}\right)}, \\
& \frac{\partial R_{C}^{(k)}}{\partial \boldsymbol{y}} \boldsymbol{d} \boldsymbol{y}^{(\boldsymbol{k})}+\frac{\partial R_{C}^{(k)}}{\partial \boldsymbol{p}} \boldsymbol{d} \boldsymbol{p}^{(\boldsymbol{k})}+\frac{\partial R_{C}^{(k)}}{\partial \boldsymbol{u}} \boldsymbol{d} \boldsymbol{u}^{(\boldsymbol{k})} \\
& -\boldsymbol{d} \boldsymbol{q}_{l}^{(k)}+\boldsymbol{d} \boldsymbol{q}_{u}^{(k)}=-R_{C}^{(k)}, \\
& \boldsymbol{q}_{l}^{(k)} \odot \boldsymbol{d} \boldsymbol{u}_{l}^{(k)}+\left(\boldsymbol{u}^{(k)}-\underline{u}\right) \odot \boldsymbol{d} \boldsymbol{q}_{l}^{(k)}=\varkappa^{(k)} \mathbb{1}-\boldsymbol{q}_{l}^{(k)} \odot\left(\boldsymbol{u}^{(k)}-\underline{u}\right), \\
& -\boldsymbol{q}_{\boldsymbol{u}}^{(k)} \odot \boldsymbol{d} \boldsymbol{u}_{\boldsymbol{u}}^{(k)}+\left(\overline{\boldsymbol{u}}-\boldsymbol{u}^{(k)}\right) \odot \boldsymbol{d} \boldsymbol{q}_{u}^{(k)}=\varkappa^{(k)} \mathbb{1}-\boldsymbol{q}_{u}^{(k)} \odot\left(\overline{\boldsymbol{u}}-\boldsymbol{u}^{(k)}\right) .
\end{aligned}
$$

(d) Compute the step length $s^{(k)}$ by using the following formula:

$$
s^{(k)}:=\min \left(1, \zeta \min \left(s_{\boldsymbol{q}_{l}}^{(k)}, s_{\boldsymbol{q}_{u}}^{(k)}, s_{\boldsymbol{u}-\underline{\boldsymbol{u}}}^{(k)}, s_{\overline{\boldsymbol{u}}-\boldsymbol{u}}^{(k)}\right)\right)
$$

with

$$
s_{\boldsymbol{q}_{l}}^{(k)}=\left\{\begin{array}{l}
\min \left(-\frac{\left(\boldsymbol{q}_{\boldsymbol{l}}^{(\boldsymbol{k})}\right)_{i}}{\left(\boldsymbol{d} \boldsymbol{q}_{l}^{(k)}\right)_{i}}: i \in \mathcal{N}_{\boldsymbol{q}_{l}}^{(k)}:=\left\{i:\left(\boldsymbol{d q}_{l}^{(k)}\right)_{i}<0\right\}\right) \text { if } \mathcal{N}_{\boldsymbol{q}_{l}}^{(k)} \neq \emptyset \\
+\infty \text { otherwise, }
\end{array}\right.
$$

and analogously for $s_{\boldsymbol{q}_{\boldsymbol{u}}}^{(k)}, s_{\boldsymbol{u}-\underline{\boldsymbol{u}}}^{(k)}$, and $s_{\overline{\boldsymbol{u}}-\boldsymbol{u}}^{(k)}$.

(e) Compute the updates

$$
\begin{aligned}
& \boldsymbol{u}^{(k+1)}=\boldsymbol{u}^{(k)}+s^{(k)} \boldsymbol{d} \boldsymbol{u}^{(k)}, \\
& \boldsymbol{p}^{(k+1)}=\boldsymbol{p}^{(k)}+s^{(k)} \boldsymbol{d} \boldsymbol{p}^{(k)}, \\
& \boldsymbol{q}_{\boldsymbol{l}}^{(k+1)}=\boldsymbol{q}_{\boldsymbol{l}}^{(k)}+s^{(k)} \boldsymbol{d} \boldsymbol{q}_{l}^{(k)}, \\
& \boldsymbol{q}_{\boldsymbol{u}}^{(k+1)}=\boldsymbol{q}_{\boldsymbol{u}}^{(k)}+s^{(k)} \boldsymbol{d} \boldsymbol{q}_{\boldsymbol{u}}^{(k)}, \\
& \varkappa^{(k+1)}=\epsilon \varkappa^{(k)} .
\end{aligned}
$$

(3) Return to (2).

For a complete discussion of the algorithm and its properties, we refer to [21]. In fact, it turns out that the algorithm is a particular realization of an implicit programming approach to the discrete version of $\left(\mathrm{P}_{0}\right)$. Here we very briefly address the main features of the algorithm: 
Step (1). This is the classical initialization in the context of interior point methods, where $\boldsymbol{u}^{(0)}$ should be chosen safely away from the bounds. The bounds may only be approached as $\varkappa^{(k)} \downarrow 0$. The particular form of $\varkappa^{(0)}$ takes into account the mismatch in complementarity of the initial $\boldsymbol{u}^{(0)}, \boldsymbol{q}_{l}^{(0)}$, and $\boldsymbol{q}_{\boldsymbol{u}}^{(\mathbf{0})}$. If the initial $\varkappa$-value is too small, the update steps force $\boldsymbol{u}^{(\boldsymbol{k})}$ close to the bounds already early along the iterations. This would typically have an adverse effect on the convergence speed of the algorithm.

Step (2a) corresponds to a feasibility restoration yielding $\left(\boldsymbol{y}^{(\boldsymbol{k})}, \boldsymbol{u}^{(\boldsymbol{k})}, \boldsymbol{\xi}^{(\boldsymbol{k})}\right)$; i.e., given $\boldsymbol{u}^{(\boldsymbol{k})}$, a feasible pair $\left(\boldsymbol{y}^{(\boldsymbol{k})}, \boldsymbol{\xi}^{(\boldsymbol{k})}\right)$ is computed satisfying the Black-Scholes model. For the numerical solution of the Black-Scholes variational inequality with a given volatility $\boldsymbol{u}^{(k)}$, a semismooth Newton solver may be used; see [24] and the references therein for a general account of semismooth Newton methods together with their local convergence properties. This solution technique relies on a complementarity function-based reformulation of

$$
\boldsymbol{y} \geq 0, \quad \boldsymbol{\xi} \geq 0, \quad \boldsymbol{y} \odot \boldsymbol{\xi}=0,
$$

and its "linearization" using an appropriately chosen generalized derivative. In particular, the above complementarity system is equivalent to

$$
\boldsymbol{\Theta}(\boldsymbol{y}, \boldsymbol{\xi})=\left(\Theta\left(\boldsymbol{y}_{1}, \boldsymbol{\xi}_{1}\right), \ldots, \Theta\left(\boldsymbol{y}_{N_{y}}, \boldsymbol{\xi}_{N_{y}}\right)\right)=0,
$$

where $\Theta(a, b)=a-\max (0, a-\varsigma b)$ for $a, b \in \mathbb{R}$ and a fixed $\varsigma>0$ represents a particular choice of a complementarity function. For setting up the Newton system in every step, a "linearization" of the max-term has to be computed (note that the first equation in (2a) involving $R_{S}$ is continuously differentiable, and hence can be treated in the standard way). This can be done by using the generalized derivative of [24] resulting in

$$
\partial \max (0, \boldsymbol{z})=\operatorname{diag}\left(d_{1}, \ldots, d_{N_{y}}\right) \quad \text { with } d_{i}= \begin{cases}0 & \text { if } \boldsymbol{z}_{i} \leq 0 \\ 1 & \text { if } \boldsymbol{z}_{i}>0\end{cases}
$$

for $\boldsymbol{z} \in \mathbb{R}^{N_{y}}$.

Step (2b). Since $\mathcal{A S}\left(\boldsymbol{y}^{\star}\right)$ and $\mathcal{I} \mathcal{S}\left(\boldsymbol{y}^{\star}\right)$ are unknown during the iteration, these sets are estimated based on the current iterate and feasible point $\left(\boldsymbol{y}^{(k)}, \boldsymbol{u}^{(k)}, \boldsymbol{\xi}^{(k)}\right)$.

Step (2c). The update step is computed by solving the linearization of

$$
\begin{aligned}
R_{S}(\boldsymbol{y}, \boldsymbol{u})_{i} & =0, \quad i \in \mathcal{I S}(\boldsymbol{y}), \\
\boldsymbol{y}_{i} & =0, \quad i \in \mathcal{A S}(\boldsymbol{y}), \\
R_{A}(\boldsymbol{y}, \boldsymbol{p}, \boldsymbol{u})_{i} & =0, \quad i \in \mathcal{I S}(\boldsymbol{y}), \\
\boldsymbol{p}_{i} & =0, \quad i \in \mathcal{A S}(\boldsymbol{y}), \\
R_{C}(\boldsymbol{y}, \boldsymbol{p}, \boldsymbol{u})-\boldsymbol{q}_{\boldsymbol{l}}+\boldsymbol{q}_{\boldsymbol{u}} & =0, \\
\boldsymbol{q}_{\boldsymbol{l}} \odot(\boldsymbol{u}-\underline{\boldsymbol{u}})-\varkappa \mathbb{1} & =0, \\
\boldsymbol{q}_{\boldsymbol{u}} \odot(\overline{\boldsymbol{u}}-\boldsymbol{u})-\varkappa \mathbb{1} & =0,
\end{aligned}
$$

at $\left(\boldsymbol{y}^{(k)}, \boldsymbol{p}^{(k)}, \boldsymbol{u}^{(k)}, \boldsymbol{q}_{l}^{(k)}, \boldsymbol{q}_{u}^{(k)}\right)$. Note that for indices $i \in \mathcal{A S}\left(\boldsymbol{y}^{(k)}\right)$ we expect $\boldsymbol{y}_{i}^{(k+1)}=$ 0 . Hence, since $\boldsymbol{y}_{i}^{(\boldsymbol{k})}=0$ for $i \in \mathcal{A S}\left(\boldsymbol{y}^{(\boldsymbol{k})}\right)$, the linearization becomes $\boldsymbol{d} \boldsymbol{y}_{i}^{(\boldsymbol{k})}=0$ for $i \in \mathcal{A S}\left(\boldsymbol{y}^{(\boldsymbol{k})}\right)$, which corresponds to the second equation in (2c). For $i \in \mathcal{I} \mathcal{S}\left(\boldsymbol{y}^{(\boldsymbol{k})}\right)$ we use the facts that $\boldsymbol{\xi}_{i}^{(\boldsymbol{k})}=0$ and $\boldsymbol{\xi}_{i}^{(\boldsymbol{k}+\mathbf{1})}=0$. This explains the first equation in (2c). We proceed now under a strict complementarity assumption on $\boldsymbol{y}_{i}^{(\boldsymbol{k})}$ and $\boldsymbol{\xi}_{i}^{(\boldsymbol{k})}$, i.e.,

$$
\mathcal{A S}\left(\boldsymbol{y}^{(k)}\right)=\mathcal{I S}\left(\boldsymbol{\xi}^{(k)}\right)=\left\{i \in\left\{1 \cdots N_{y}\right\}: \boldsymbol{\xi}_{i}^{(k)}>0\right\} .
$$


Note that under this assumption we have $\boldsymbol{p}_{i}^{(\boldsymbol{k}+\mathbf{1})}=0$ for all $i \in \mathcal{A S}\left(\boldsymbol{y}^{(\boldsymbol{k})}\right)$, and hence $\boldsymbol{d}_{i}^{(\boldsymbol{k})}=-\boldsymbol{p}_{i}^{(\boldsymbol{k})}$ for $i \in \mathcal{A} \mathcal{S}\left(\boldsymbol{y}^{(k)}\right)$, which corresponds to the fourth equation in (2c). The remaining equations in (2c) are obtained by a straightforward linearization.

Step (2d) reflects the fraction-to-the-boundary rule from interior point methods. The step length computed here guarantees

$$
\underline{\boldsymbol{u}}_{i}<\boldsymbol{u}_{i}^{(\boldsymbol{k}+\mathbf{1})}<\overline{\boldsymbol{u}}_{i}, \quad \boldsymbol{q}_{\boldsymbol{l}, \boldsymbol{i}}^{(\boldsymbol{k}+\mathbf{1})}>0, \quad \boldsymbol{q}_{\boldsymbol{u}, i}^{(\boldsymbol{k}+\mathbf{1})}>0 \quad \forall i \in\left\{1 \cdots N_{u}\right\} .
$$

Notice that upon termination in step 2 and setting $\boldsymbol{\vartheta}_{i}^{(\boldsymbol{k}+\mathbf{1})}=0$ for all $i \in \mathcal{I} \mathcal{S}\left(\boldsymbol{y}^{(\boldsymbol{k})}\right)$ and $\boldsymbol{\vartheta}_{i}^{(\boldsymbol{k}+\mathbf{1})}=\left(R_{A}^{(k+1)}\right)_{i}$ for all $i \in \mathcal{A S}\left(\boldsymbol{y}^{(\boldsymbol{k})}\right)$, the final iterate $\left(\boldsymbol{y}^{(\boldsymbol{k}+\mathbf{1})}, \boldsymbol{p}^{(\boldsymbol{k}+\mathbf{1})}, \boldsymbol{\xi}^{(\boldsymbol{k})}\right.$, $\left.\vartheta^{(k+1)}\right)$ satisfies

$$
\begin{aligned}
y^{(k+1)} \odot \vartheta^{(k+1)} & =0 \\
p^{(k+1)} \odot \xi^{(k)} & =0 \\
p^{(k+1)} \odot \vartheta^{(k+1)} & \leq 0
\end{aligned}
$$

7. Numerical results. Before we describe the particular discretization used and the details of the employed solvers, we first address some structural issues.

In applications it turns out that the singular set

$$
\mathcal{S}_{0}:=\left\{(t, x) \in Q: \frac{\partial^{2} y(t, x)}{\partial x^{2}} \approx 0\right\}
$$

may cover a significant part of the overall time-space cylinder. On this set (and in particular on $\left\{(t, x): \frac{\partial^{2} y(t, x)}{\partial x^{2}}=0\right\}$ ) a reconstruction of $u$ is impossible unless a priori information is used. In fact, here we assume that a reference parameter $u_{\text {ref }}$ is known such that $u_{\text {ref }} \mid \mathcal{S}_{0}$ represents a good approximation of the true parameter. Then the set $\mathcal{S}_{0}$ is estimated from the given data $y_{d}$, and a penalty term

$$
J_{\mathrm{ref}}(u)=\int_{0}^{T} \int_{\Omega} \nu_{\mathcal{S}_{0}}(t, x)\left(u(t, x)-u_{\text {ref }}(t, x)\right)^{2} d x d t
$$

is added to $J(y, u)$ in order to keep $u$ close to $u_{\text {ref. }}$. The latter is achieved by an appropriate choice of the penalty parameter $\nu_{\mathcal{S}_{0}}=\nu_{\mathcal{S}_{0}}(t, x)$, which is assumed to be sufficiently large in $\mathcal{S}_{0}$ and small outside.

In the test example below we fix the reference parameter $u_{\text {ref }}\left(=0.02 x^{2}\right)$ and introduce the constraints

$$
\frac{1}{10} \min _{Q}\left(u_{r e f}(t, x)\right) \leq u(t, x) \leq 10 \max _{Q}\left(u_{r e f}(t, x)\right)
$$

in coherence with the theory. We point out that none of the bounds on $u$ is active at the solution upon termination of our algorithm.

For a fixed reference value $u$, the data $y_{d}$ is obtained by solving the Black-Scholes model on a fine mesh and then projecting the solution onto the computational (coarse) mesh. In our tests, the data without noise is used for estimating $\mathcal{S}_{0}$ (red region in the computational domain (cf. Figure 7.1)); otherwise, i.e., in the presence of (Gaussian) noise, (local Gaussian) smoothing has to be applied before estimating the singular set. 

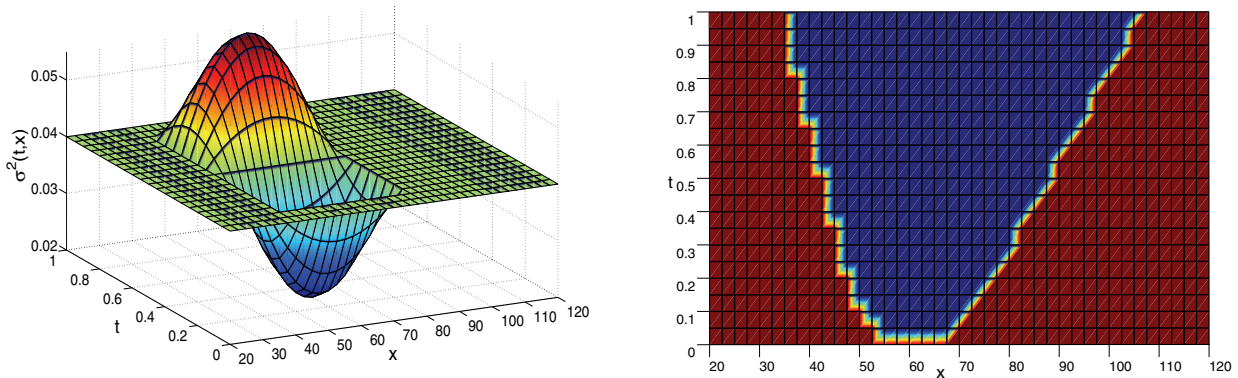

FIG. 7.1. Exact squared volatility (left) and computational domain (right).

All results below are based on the domain $Q=(0, T) \times \Omega:=(0,1) \times(20,120)$ and the strike price $K=60$. Our goal is to identify the volatility whose square is plotted in Figure 7.1 and expressed by

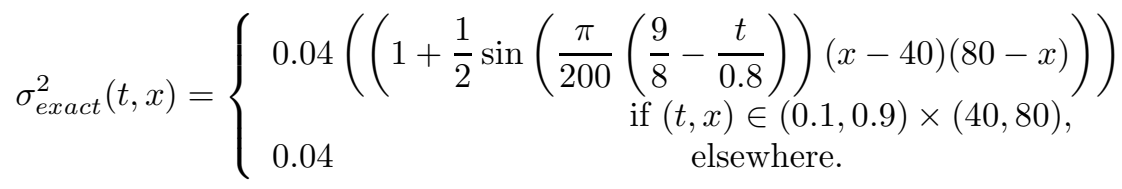

We recall that

$$
\begin{gathered}
u(t, x)=\frac{1}{2} x^{2} \sigma^{2}(t, x), \\
y(t, x)=e^{-\tau t}\left(P(t, x)-P_{0}(t, x)\right)
\end{gathered}
$$

for some $\tau>0$.

For the discretization of the first-order system, we use an implicit Euler scheme in time and finite elements in space. According to the $H^{2}(\Omega)$-regularity requirement for the continuous volatility, we use Hermite elements for discretizing $u$ and use continuous piecewise linear finite elements for discretizing the quantities $y$ and $p$ in space, respectively. We point out that the discretizations of the multipliers $\xi$ and $\vartheta$ result from the discretizations of $y, p$, and $u$, and similarly for $q_{l}$ and $q_{u}$. All results reported below are based on a $20 \times 40$-uniform mesh in the time-space domain. The data $y_{d}$ is computed by first solving the Black-Scholes variational inequality for $\sigma=\sigma_{\text {exact }}$ on a $50 \times 100$ mesh and then projecting onto the underlying $20 \times 40$ mesh. Finally, we consider measurements with $0 \%, 0.1 \%$, and $2 \%$ Gaussian white noise; see Figures 7.2-7.4(c).

In the feasibility restoration step (2a) of our algorithm we use a semismooth Newton solver, which we implement in its equivalent primal-dual active-set form [24]. The linear system of step (2c) is solved by the biconjugate gradient stabilized (BICGSTAB) method with incomplete LU (ILU) preconditioning.

We initialize the algorithm by

$$
\mathbf{q}_{\mathbf{l}}^{(\mathbf{0})}=10, \quad \mathbf{q}_{\mathbf{u}}^{(\mathbf{0})}=10, \quad \mathbf{p}^{(\mathbf{0})}=0, \quad \mathbf{u}^{(\mathbf{0})}=u_{\mathrm{ref}}, \quad \epsilon=0.6, \quad \zeta=0.99
$$


(a)
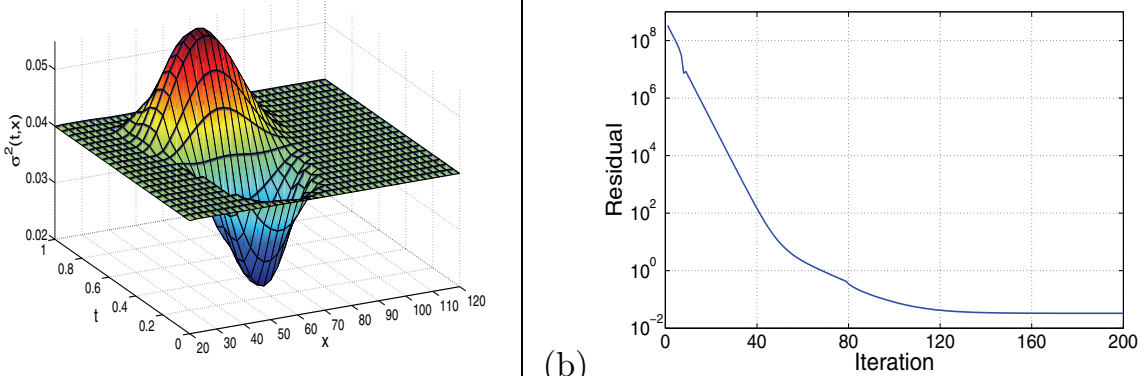

(d)

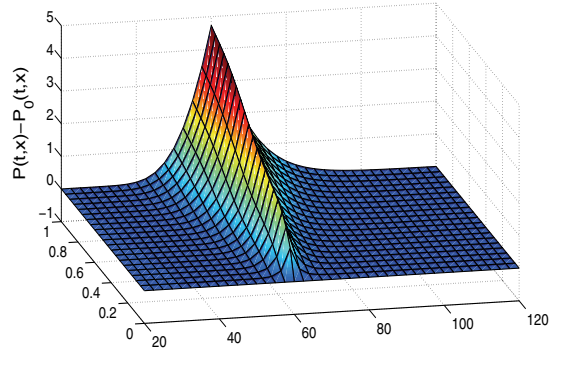

FIG. 7.2. $0 \%$ noise. (a) Computed squared volatility. (b) Convergence of residual. (c) Observed state $=$ difference between observed price and payoff function. $(\mathrm{d})$ Computed state $=$ difference between computed price and payoff function.

(a)

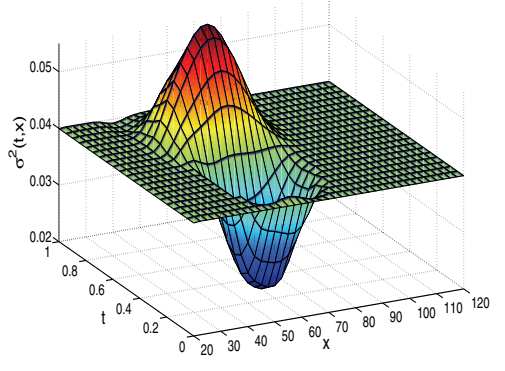

(b)

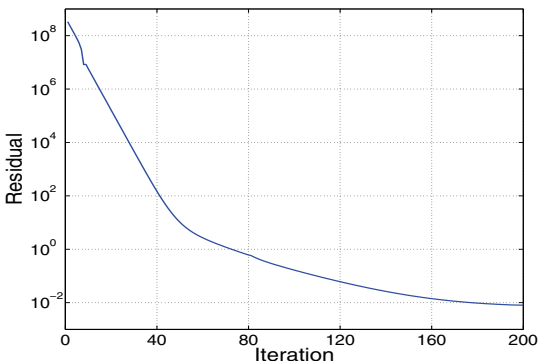

(d)

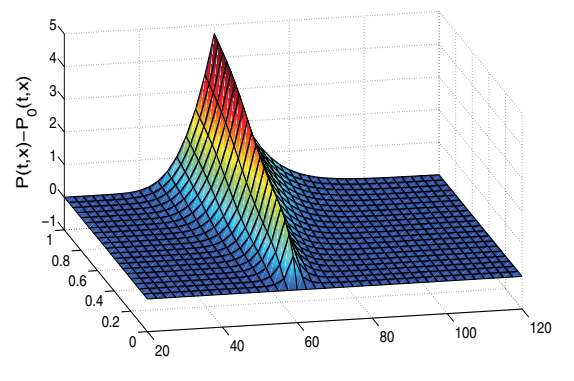

(c)

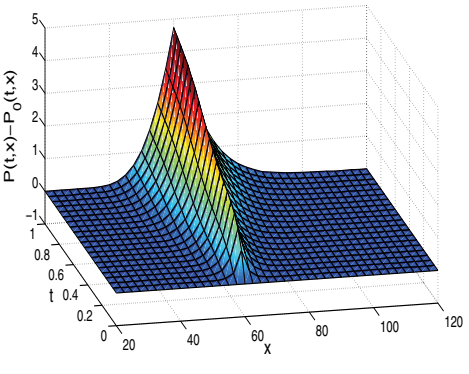

FIG. 7.3. $0.1 \%$ noise. (a) Computed squared volatility. (b) Convergence of residual. (c) Observed state $=$ difference between observed price and payoff function. (d) Computed state = difference between computed price and payoff function. 


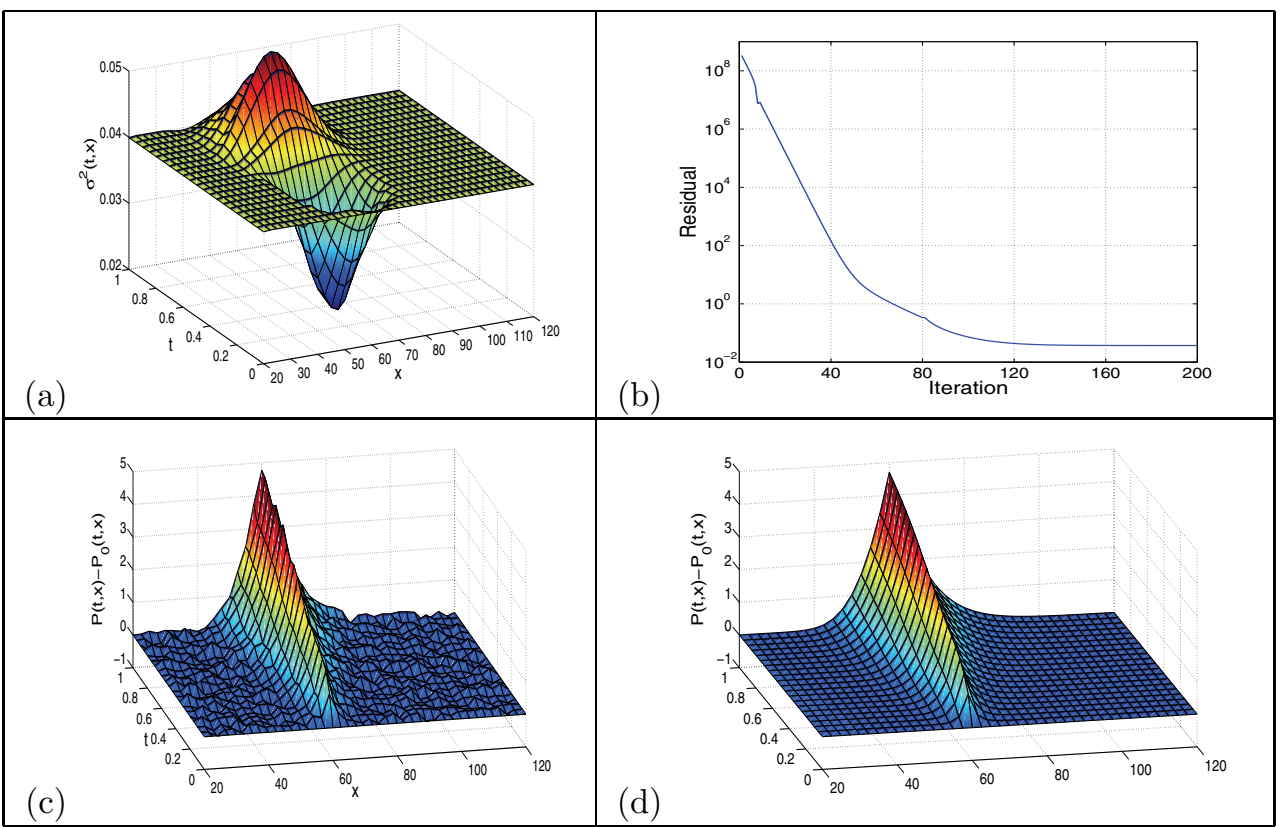

FIG. 7.4. $2 \%$ noise. (a) Computed squared volatility. (b) Convergence of residual. (c) Observed state $=$ difference between observed price and payoff function. $(\mathrm{d})$ Computed state $=$ difference between computed price and payoff function.

For tuning purposes, we replace $\frac{\nu}{2}\|u\|_{\mathcal{U}}^{2}$ in the cost function by $\frac{1}{2}\|u\|_{\mathcal{U}, \nu}^{2}$ with $\|u\|_{\mathcal{U}, \nu}^{2}=$ $\langle u, u\rangle_{\mathcal{U}, \nu}$ and

$$
\begin{aligned}
& \langle u, v\rangle_{\mathcal{U}, \nu}=\quad \nu_{00}(u, v)_{L^{2}(Q)} \\
& +\nu_{10}\left(\frac{\partial u}{\partial t}, \frac{\partial v}{\partial t}\right)_{L^{2}(Q)} \\
& +\nu_{01}\left(\frac{\partial u}{\partial x}, \frac{\partial v}{\partial x}\right)_{L^{2}(Q)}+\nu_{11}\left(\frac{\partial^{2} u}{\partial t \partial x}, \frac{\partial^{2} v}{\partial t \partial x}\right)_{L^{2}(Q)} \\
& +\nu_{02}\left(\frac{\partial^{2} u}{\partial x^{2}}, \frac{\partial^{2} v}{\partial x^{2}}\right)_{L^{2}(Q)}+\nu_{12}\left(\frac{\partial^{3} u}{\partial t \partial x^{2}}, \frac{\partial^{3} v}{\partial t \partial x^{2}}\right)_{L^{2}(Q)} .
\end{aligned}
$$

The values of the weights in (7.1) are summarized in Table 7.1.

TABLE 7.1

Weight and regularization parameters.

\begin{tabular}{cccc}
\hline Noise & $0 \%$ & $0.1 \%$ & $2 \%$ \\
\hline$\nu_{00}$ & $1 \mathrm{e}-8$ & $1 \mathrm{e}-8$ & $1 \mathrm{e}-8$ \\
\hline$\nu_{01}$ & $1 \mathrm{e}-8$ & $1 \mathrm{e}-8$ & $1 \mathrm{e}-8$ \\
\hline$\nu_{02}$ & 1 & $1 \mathrm{e}-2$ & 1 \\
\hline$\nu_{10}$ & $1 \mathrm{e}-8$ & $1 \mathrm{e}-8$ & $1 \mathrm{e}-8$ \\
\hline$\nu_{11}$ & $1 \mathrm{e}-8$ & $1 \mathrm{e}-8$ & $1 \mathrm{e}-8$ \\
\hline$\nu_{12}$ & $1 \mathrm{e}-8$ & $5 \times 1 \mathrm{e}-4$ & $1 \mathrm{e}-2$ \\
\hline
\end{tabular}

We stop the algorithm after $k_{\max }=200$ iterations. In Figures $7.2-7.4$ we depict, for each noise level, the recovered squared volatility, its corresponding state variable, the observed state, as well as a monitor of the norm of the residuals of (6.1)-(6.6). 
Concerning our numerical results, it can be seen from Figures 7.2-7.4(b) that the residuals are reduced at least by 9 or more digits, and the recovered squared volatilities (cf. Figures 7.2-7.4(a)) are close to the true squared volatility (cf. Figure 7.1). As one would expect, the quality of the reconstruction improves with decreasing noise level. Provided we have an appropriate tuning of the numerical parameters of the algorithm, the behavior of the algorithm is stable with respect to the noise. This stability concerns the quality of the obtained reconstruction, the number of iterations, and the reduction of the residual norm. In particular, the choice of the regularization coefficients is crucial to balance the smoothing of the solution and the fidelity with respect to the observed state. Notice that we regularize mainly by the second-order term in space of the $H^{1}\left(0, T, H^{2}(\Omega)\right)$-norm; see Table 7.1. This type of tuning is the result of numerous experiments. Certainly, an automated choice of the regularization coefficients is of interest, but it may be difficult to achieve rigorously. The coefficient $\epsilon$, which is used to update the interior point parameter $\varkappa$, should be carefully chosen. The $\epsilon$-value reported above is the smallest we found such that the algorithm found a stable reconstruction. In fact for smaller values, the iterate at step $k$ gives rise to a poor initialization for the step $k+1$. Large values of $\epsilon$ result in slow convergence. We further point out that during the feasibility restoration step (2a), thanks to the interior point treatment, the matrix of the discrete variational inequality remains positive definite during the iterations, and the semismooth Newton method converges in at most 10 iterations.

\section{Extensions.}

8.1. Cost functional. The analysis carried out in the previous sections can be generalized easily to cost functionals of the form

$$
\mathcal{J}^{\delta}(y, u):=\frac{1}{2 \delta^{2}} \int_{T-\delta}^{T} \int_{x_{o b s}-\delta / 2}^{x_{o b s}+\delta / 2}\left(y-y_{d}\right)^{2} d x d t+\frac{\nu}{2}\|u\|_{\mathcal{U}}^{2}
$$

with $\delta>0$ a fixed constant, $\left.x_{o b s} \in\right] \underline{x}, \bar{x}$, and $y_{d} \in \mathbb{R}$. In particular, for a small value of $\delta, \mathcal{J}^{\delta}$ can be considered for the local volatility estimation problem from a single observation via the approximation $\left(y\left(T, x_{o b s}\right)-y_{d}\right)^{2} \approx \frac{1}{\delta^{2}} \int_{T-\delta}^{T} \int_{x_{o b s}-\delta / 2}^{x_{o b s}+\delta / 2}\left(y-y_{d}\right)^{2} d x d t$. An extension to the cost functional

$$
\mathcal{J}^{0}(y, u):=\frac{1}{2}\left(y\left(T, x_{o b s}\right)-y_{d}\right)^{2}+\frac{\nu}{2}\|u\|_{\mathcal{U}}^{2}
$$

however, requires a more regular function space setting. Indeed, in order to ensure that $\mathcal{J}^{0}$ is well defined, the space of state variables, denoted by $\mathcal{Y}$, should be at least embedded in $\mathcal{C}^{0}(\bar{Q})$. Now, regarding the regularity of $P$ in Theorem 2.2, the natural choice for $\mathcal{Y}$ appears to be

$$
\mathcal{Y}=\left\{v \in L^{2}\left(0, T, H^{2}(\Omega)\right): \frac{\partial v}{\partial t} \in L^{2}\left(0, T, L^{2}(\Omega)\right) \text { and }\left.v\right|_{\Gamma \times(0, T)}=0\right\}
$$

Notice that in this case $\mathcal{Y}$ is embedded continuously in $\mathcal{C}^{0}(\bar{Q})$ and compactly in 
$L^{2}\left(0, T, H^{1}(\Omega)\right)$ (see, for instance, $[17,38]$ ). The corresponding optimal control problem reads

$$
\left(\mathrm{P}_{\mathcal{J}^{0}}\right) \quad \begin{cases}\min & \mathcal{J}^{0}(y, u) \\ \text { over } & (y, u, \xi) \in \mathcal{Y} \times U_{a d} \times L^{2}(Q) \\ \text { s.t. } & \frac{\partial y}{\partial t}+\mathcal{A}(u) y-\xi=0 \text { in } L^{2}(Q), \\ & y(0)=y_{0} \text { in } \Omega \\ & y \geq \Psi \text { in } Q, \quad \xi \geq 0 \text { a.e. in } Q, \quad(\xi, y-\Psi)_{L^{2}(Q)}=0 .\end{cases}
$$

Here we applied the transformations

$$
\begin{aligned}
\Psi(t, x) & =e^{-\tau t}\left(P_{0}(x)-l(x)\right), \\
y_{0}(x) & =P_{0}(x)-l(x), \\
y(t, x) & =e^{-\tau t}(P(t, x)-l(x))
\end{aligned}
$$

to the original system $(2.1)-(2.7)$ with $l(x)=\frac{(K-\underline{x})}{(\underline{x}-\bar{x})}(x-\bar{x})$. The penalized-relaxed version of $\left(\mathrm{P}_{\mathcal{J}^{0}}\right)$ is defined as follows:

$$
\left(\mathrm{P}_{\mathcal{J}_{\gamma}}\right) \quad\left\{\begin{aligned}
& \min \quad \mathcal{J}_{\gamma}^{0}(y, u)=\mathcal{J}^{0}(y, u)+\frac{1}{2 \gamma}\|\max (0, \bar{\lambda}-\gamma(y-\Psi))\|_{L^{2}(\Omega)}^{2} \\
& \text { over } \quad(y, u, \xi) \in \mathcal{Y} \times U_{a d} \times L^{2}(Q) \\
& \text { s.t. } \quad \frac{\partial y}{\partial t}+\mathcal{A}(u) y-\xi=0 \quad \text { in } L^{2}(Q), \\
& y(0)=y_{0} \text { in } \Omega, \\
& \xi \geq 0 \text { a.e. in } Q, \quad(\xi, y-\Psi)_{L^{2}(Q)} \leq \alpha_{\gamma}, \\
& R \geq \frac{1}{2}\|\xi\|_{L^{2}(Q)}^{2} .
\end{aligned}\right.
$$

Applying similar arguments as in the previous sections, the following result for global solutions of $\left(\mathrm{P}_{\mathcal{J}_{\gamma}}\right)$ can be shown.

Proposition 8.1. For every $\gamma>0$, the penalized-relaxed problem $\left(\mathrm{P}_{\mathcal{J}_{\gamma}}\right)$ possesses at least one solution $\left(y_{\gamma}, u_{\gamma}, \xi_{\gamma}\right)$. Furthermore, there exist $\left(y^{*}, u^{*}, \xi^{*}\right)$ in $\mathcal{Y} \times$ $\mathcal{U} \times L^{2}(Q)$ and a subsequence, still denoted by $\left(y_{\gamma}, u_{\gamma}, \xi_{\gamma}\right)_{\gamma>0}$, such that

$$
\begin{array}{ll}
u_{\gamma} \rightarrow u^{*} & \text { in } \mathcal{U}, \\
\xi_{\gamma} \rightarrow \xi^{*} & \text { in } L^{2}(Q), \\
y_{\gamma} \rightarrow y^{*} & \text { in } \mathcal{Y}, \\
u_{\gamma} \longrightarrow u^{*} & \text { in } L^{\infty}\left(0, T, W^{1, \infty}(\Omega)\right), \\
y_{\gamma} \longrightarrow y^{*} & \text { in } L^{2}\left(0, T, H^{1}(\Omega)\right),
\end{array}
$$

and $\left(y^{*}, u^{*}, \xi^{*}\right)$ is a solution of $\left(\mathrm{P}_{\mathcal{J}^{0}}\right)$.

Furthermore one can derive the first order optimality system for the penalizedrelaxed problem.

Proposition 8.2. Let $\left(y_{\gamma}, u_{\gamma}, \xi_{\gamma}\right)$ be a solution of the problem $\left(\mathrm{P}_{\mathcal{J}_{\gamma}}\right)$. Then there exists a Lagrange multiplier vector $\left(p_{\gamma}, \mu_{\gamma}, r_{\gamma}, \kappa_{\gamma}\right)$ in $\mathcal{Y} \times L^{2}(Q) \times \mathbb{R} \times \mathbb{R}$ such that the following first order optimality system holds true:

$$
\begin{aligned}
\frac{\partial y_{\gamma}}{\partial t}+\mathcal{A}\left(u_{\gamma}\right) y_{\gamma}-\xi_{\gamma} & =0 \quad \text { in } L^{2}(Q), \\
y_{\gamma}(0) & =y_{0} \quad \text { in } \Omega,
\end{aligned}
$$

Copyright $\odot$ by SIAM. Unauthorized reproduction of this article is prohibited. 


$$
\begin{aligned}
& \left(\frac{\partial v}{\partial t}, p_{\gamma}\right)_{L^{2}(Q)}+\left(\mathcal{A}\left(u_{\gamma}\right) v, p_{\gamma}\right)_{L^{2}(Q)}=\left(y\left(T, x_{o b s}\right)-y_{d}\right) v\left(T, x_{o b s}\right) \\
& +\left(r_{\gamma} \xi_{\gamma}, v\right)_{L^{2}(Q)}-\left(\max \left(0, \bar{\lambda}-\gamma\left(y_{\gamma}-\Psi\right)\right), v\right)_{L^{2}(Q)} \quad \forall v \in \mathcal{Y}, \\
& \nu\left\langle u_{\gamma}, u-u_{\gamma}\right\rangle_{\mathcal{U}}-\left(\mathcal{A}^{\prime}\left(u-u_{\gamma}\right) y_{\gamma}, p_{\gamma}\right)_{L^{2}(Q)} \geq 0 \quad \forall u \in U_{a d}, \\
& \kappa_{\gamma} \xi_{\gamma}+p_{\gamma}-\mu_{\gamma}+r_{\gamma}\left(y_{\gamma}-\Psi\right)=0 \quad \text { a.e. in } Q \text {, } \\
& \xi_{\gamma} \geq 0 \text { a.e. in } Q, \quad \mu_{\gamma} \geq 0 \text { a.e. in } Q,\left(\xi_{\gamma}, \mu_{\gamma}\right)_{L^{2}(Q)}=0 \text {, } \\
& \kappa_{\gamma} \geq 0, \quad \frac{1}{2}\left\|\xi_{\gamma}\right\|_{L^{2}(Q)}^{2} \leq R, \quad \frac{\kappa_{\gamma}}{2}\left\|\xi_{\gamma}\right\|_{L^{2}(Q)}^{2}=\kappa_{\gamma} R, \\
& r_{\gamma} \geq 0, \quad\left(y_{\gamma}-\Psi, \xi_{\gamma}\right)_{L^{2}(Q)} \leq \alpha_{\gamma}, \quad r_{\gamma}\left(y_{\gamma}-\Psi, \xi_{\gamma}\right)_{L^{2}(Q)}=r_{\gamma} \alpha_{\gamma} .
\end{aligned}
$$

Investigating the limit of the previous optimality system as $\gamma \rightarrow \infty$ requires a more involved analysis than the one of section 5. In particular, the boundedness of the sequence of adjoint states $\left(p_{\gamma}\right)$ is a challenge. In fact, in contrast to techniques relying on penalization schemes (see, e.g., [1]), which appear to lead to weaker types of stationarity, one has to overcome the difficulty due to the lack of monotonicity in the adjoint equation in order to obtain a (uniform in $\gamma$ ) bound on $\left(p_{\gamma}\right)$. The discrete version of (8.10)-(8.17), however, leads to an algebraic system of a form similar to (6.1)-(6.5) when $\gamma \rightarrow \infty$ such that our numerical approach of section 6 carries over to this case. The extension of the analysis to the case of an observation of multiple prices with different maturities and different strikes follows immediately from a single observation case. For a related approach see [1]. Numerically, our proposed algorithm might be used in an iterative way to solve the calibration problem which will be the subject of our future work.

8.2. Differential operator. An extension to optimal control problems with more general differential operators $\mathcal{A}$ is also possible as far as the properties required in Lemma 2.1 hold true within an appropriate function space setting. For instance, it is straightforward to obtain a similar optimality system as in Theorem 5.2 for an optimal control problem governed by a parabolic variational inequality with a divergence form operator:

$$
\begin{cases}\min & J(y, u)=\frac{1}{2}\left\|y-y_{d}\right\|_{L^{2}(Q)}^{2}+\frac{1}{2}\left\|y(T, \cdot)-y_{T}\right\|_{L^{2}(\Omega)}^{2}+\frac{\nu}{2}\|u\|_{\mathcal{U}}^{2} \\ \text { over } & (y, u) \in W(0, T) \times \mathcal{U} \\ \text { s.t. } & u \in U_{a d}=\{u \in \mathcal{U}: 0<\underline{u} \leq u \leq \bar{u} \text { a.e. in } Q\} \\ & \left\langle\frac{\partial y}{\partial t}-\operatorname{div}(u \nabla y)-f, v-y\right\rangle \geq 0 \forall v \in L^{2}\left(0, T, H_{0}^{1}(\Omega)\right) \text { with } v \geq 0 \text { a.e. in } Q, \\ & y \geq 0 \text { a.e. in } Q \\ & y(0)=0 .\end{cases}
$$

In this case, one may consider the larger control space

$$
\mathcal{U}=\mathcal{W}:=\left\{u \in L^{2}(Q): \frac{\partial u}{\partial x}, \frac{\partial u}{\partial t}, \frac{\partial^{2} u}{\partial t \partial x} \in L^{2}(Q)\right\}
$$

since the derivative of $u$ with respect to time is not involved.

For a complete analytical and numerical study of $\left(\mathrm{P}_{d i v}\right)$ taking the right-hand side $f$ as a control variable and a second-order linear elliptic partial differential operator with respect to space $x$, which is independent of $u$, in the state system, we refer to [27]. 


\section{REFERENCES}

[1] Y. AchDou, An inverse problem for a parabolic variational inequality arising in votality calibration with American options, SIAM J. Control Optim., 43 (2005), pp. 1583-1615.

[2] Y. AcHDOU, An inverse problem for a parabolic variational inequality with an integrodifferential operator, SIAM J. Control Optim., 47 (2008), pp. 733-767.

[3] Y. Achdou And O. Pironneau, Volatility smile by multilevel least square, Int. J. Theor. Appl. Finance, 5 (2002), pp. 619-643.

[4] Y. Achdou and O. Pironneau, A numerical procedure for the calibration of volatility with American options, Appl. Math. Finance, 12 (2005), pp. 201-241.

[5] Y. Achdou and O. Pironneau, Computational Methods for Option Pricing, Frontiers Appl. Math. 30, SIAM, Philadelphia, 2005.

[6] M. Avellaneda, C. Friedman, R. Holmes, and D. Samperi, Calibrating volatility surfaces via relative-entropy minimization, Appl. Math. Finance, 41 (1997), pp. 37-64.

[7] H. Berestycki, J. Busca, and I. Florent, Asymptotics and calibration of local volatility models, Quant. Finance, 2 (2002), pp. 61-69.

[8] M. Bergounioux, Optimal control of an obstacle problem, Appl. Math. Optim., 36 (1997), pp. $147-172$.

[9] F. Black and M. Scholes, The pricing of options and corporate liabilities, J. Political Economy, 81 (1973), pp. 637-654.

[10] J. Bodurtha AND M. Jermakyan, Nonparametric estimation of an implied volatility surface, J. Comput. Finance, 2 (1999), pp. 29-60.

[11] I. BOUChOUEV AND V. IsAKOV, Uniqueness, stability and numerical methods for the inverse problem that arises in financial markets, Inverse Problems, 15 (1999), pp. 95-116.

[12] T. Coleman, Y. Li, And A. Verma, Reconstructing the unknown volatility function, J. Comput. Finance, 2 (1999), pp. 77-102.

[13] S. CRÉPEY, Calibration of the local volatility in a trinomial tree using Tikhonov regularization, Inverse Problems, 19 (2003), pp. 91-127.

[14] S. CRÉPEY, Calibration of the local volatility in a generalized Black-Scholes model using Tikhonov regularization, SIAM J. Math. Anal., 34 (2003), pp. 1183-1206.

[15] B. DupIRE, Pricing with smile, Risk, 7 (1994), pp. 18-20.

[16] B. DürInG, Black-Scholes Type Equations: Mathematical Analysis, Parameter Identification and Numerical Solution, Ph.D. thesis, J. Gutenberg-Universität Mainz, Mainz, Germany, 2005. Available online at http://ubm.opus.hbz-nrw.de/volltexte/2005/891/pdf/diss.pdf.

[17] L. C. Evans, Partial Differential Equations, American Mathematical Society, Providence, RI, 1998.

[18] M. Hanke And E. Rösler, Computation of local volatilities from regularized Dupire equations, Int. J. Theor. Appl. Finance, 8 (2005), pp. 207-222.

[19] H. EgGer And H. W. Engl, Tikhonov regularization applied to the inverse problem of option pricing: Convergence analysis and rates, Inverse Problems, 21 (2005), pp. 1027-1045.

[20] M. Hintermüller, Inverse coefficient problems for variational inequalities: Optimality conditions and numerical realization, M2AN Math. Model. Numer. Anal., 35 (2001), pp. 129152.

[21] M. HintermüLLER, An active-set equality constrained Newton solver with feasibility restoration for inverse coefficient problems in elliptic variational inequalities, Inverse Problems, 24 (2008), article 034017.

[22] M. Hintermüller AND I. KopackA, Mathematical programs with complementarity constraints in function space: $C$ - and strong stationarity and a path-following algorithm, SIAM J. Optim., 20 (2009), pp. 868-902.

[23] M. Hintermüller AND K. Kunisch, Path-following methods for a class of constrained minimization problems in function space, SIAM J. Optim., 17 (2006), pp. 159-187.

[24] M. Hintermüller, K. Ito, AND K. Kunisch, The primal-dual active set strategy as a semismooth Newton method, SIAM J. Optim., 13 (2003), pp. 865-888.

[25] K. Iто And K. Kunisch, Parabolic variational inequalities: The Lagrange multiplier approach, J. Math. Pures Appl., 85 (2006), pp. 415-449.

[26] R. Kangro and R. Nicolaides, Far field boundary conditions for Black-Scholes equations, SIAM J. Numer. Anal., 38 (2000), pp. 1357-1368.

[27] I. Kopacka, MPECs/MPCCs in Function Space: First Order Optimality Concepts, PathFollowing, and Multilevel Algorithms, Ph.D. Thesis, Institut für Mathematik und Wissenschaftliches Rechnen, University of Graz, Graz, Austria, 2009.

[28] K. Kunisch And G. Peichl, Estimation of a Temporally and Spatially Varying Diffusion Coefficient in a Parabolic System by an Augmented Lagrangian Technique, Technical Report 146, University of Graz, Graz, Austria, 1989.

Copyright (c) by SIAM. Unauthorized reproduction of this article is prohibited. 
[29] K. Kunisch And G. Peichl, Estimation of a temporally and spatially varying diffusion coefficient in a parabolic system by an augmented Lagrangian technique, Numer. Math., 59 (1991), pp. 473-509.

[30] Z. Q. Luo, J. S. PANG, AND D. Ralph, Mathematical Programs with Equilibrium Constraints, Cambridge University Press, Cambridge, UK, 1996.

[31] R. C. Merton, Theory of rational option pricing, Bell J. Econom. and Management Sci., 4 (1973), pp. 141-183.

[32] R. Mignot And J.-P. Puel, Optimal control in some variational inequalities, SIAM J. Control Optim., 22 (1984), pp. 466-476.

[33] B. S. Mordukhovich, Variational Analysis and Generalized Differentiation: Basic Theory, Springer-Verlag, Berlin, 2006.

[34] B. S. Mordukhovich, Variational Analysis and Generalized Differentiation: Applications, Springer-Verlag, Berlin, 2006.

[35] R. Myneni, The pricing of the American option, Ann. Appl. Probab., 2 (1992), pp. 1-23.

[36] J. Outrata, M. Kočvara, And J. Zowe, Nonsmooth Approach to Optimization Problems with Equilibrium Constraints: Theory, Applications and Numerical Results, Kluwer, Dordrecht, 1998.

[37] H. Scheel And S. Scholtes, Mathematical programs with complementarity constraints: Stationarity, optimality and sensitivity, Math. Oper. Res., 25 (2000), pp. 1-22.

[38] R. Temam, Navier-Stokes Equations, North-Holland, Amsterdam, 1977.

[39] S. J. Wright, Primal-Dual Interior-Point Methods, SIAM, Philadelphia, 1997.

[40] P. Wilmott, J. Dewynne, and S. Howison, Option Pricing: Mathematical Models and Computation, Oxford Financial Press, Oxford, UK, 1993.

[41] J. J. YE, Necessary and sufficient optimality conditions for mathematical programs with equilibrium constraints, J. Math. Anal. Appl., 307 (2005), pp. 350-369.

[42] J. Zowe And S. KurCyusz, Regularity and stability for the mathematical programming problem in Banach spaces, Appl. Math. Optim., 5 (1979), pp. 49-62.

Copyright (C) by SIAM. Unauthorized reproduction of this article is prohibited. 